\title{
An Analytical Model for a Pneumatic Vibration Isolator with the Stiffness Effect of the Elastomeric Diaphragm
}

\author{
Dengfeng Xu $\mathbb{D}^{\mathbb{D}},{ }^{1,2}$ Qiang Yu, ${ }^{3}$ Fei Shen, ${ }^{3}$ Yu Zhu, ${ }^{1,2,3}$ and Gaofeng Guan ${ }^{3}$ \\ ${ }^{1}$ State Key Laboratory of Tribology, Department of Mechanical Engineering, Tsinghua University, Beijing 100084, China \\ ${ }^{2}$ Beijing Lab of Precision/Ultra-Precision Manufacture Equipment and Control, Tsinghua University, Beijing 100084, China \\ ${ }^{3}$ School of Mechanical and Electrical Engineering, University of Electronic Science and Technology of China, Chengdu 611731, China
}

Correspondence should be addressed to Dengfeng Xu; xudf02@mails.tsinghua.edu.cn

Received 4 April 2018; Accepted 20 May 2018; Published 5 July 2018

Academic Editor: Pedro Museros

Copyright (C) 2018 Dengfeng Xu et al. This is an open access article distributed under the Creative Commons Attribution License, which permits unrestricted use, distribution, and reproduction in any medium, provided the original work is properly cited.

\begin{abstract}
The elastomeric diaphragm is widely used in pneumatic vibration isolators, and the relevant model is often ignored due to its complexity. Considering that the ignored model of the elastomeric diaphragm in pneumatic vibration isolators plays an important role in the discrepancy between the predicted and practical behavior, this paper develops an analytical model for the elastomeric diaphragm using the Mooney-Rivlin modeling method and elastomeric theory. Specifically, the elastomeric diaphragm consists of several segments in the axial section. After considering the structural restriction, each segment can be simplified as uniaxial stretching, and the force-strain equation can be established for each segment. By combining the equations of all segments, an analytical model of the elastomeric diaphragm can be built and solved via numerical methods. The developed model is added to the standard model of pneumatic cylinders to supply a complete analytical model for pneumatic vibration isolators. The experimental results demonstrate that the analytically predicted behavior is similar to the practical behavior. The proposed analytical model can be used as a guide for the parameter design of pneumatic isolators in practice.
\end{abstract}

\section{Introduction}

Pneumatic vibration isolators are widely applied in optics, semiconductors, and other fields $[1,2]$. Many efforts have been devoted to the design of active control systems, which require an accurate mathematical model of the pneumatic vibration isolator [3, 4]. Harris [5] assumed that the airflow in the damping hole was laminar and first offered a linear model for the pneumatic vibration isolators. In 1984, Debra and Bryan [6] established a simpler linear model of the volume ratio of the upper chamber to the lower chamber and the inherent frequency. Erin and Wilson [7] proposed an analytical modeling method for the pneumatic vibration isolator based on the ideal gas state equation. In all previous studies, the discrepancies between the model-predicted behavior and the practically observed behavior of pneumatic vibration isolators exceeded $50 \%$ because the effect of the elastomeric diaphragm was ignored.
To obtain a more accurate model of the pneumatic isolator, Erin and Wilson proposed an improved model for pneumatic isolators by incorporating the stiffness and damping effect of the elastomeric diaphragm based on experimental investigation. Lee and Kim $[8,9]$ reported a nonlinear complex stiffness model that reflected the frequency and excitation amplitude, which was validated by comparison with experimental measurements. Pu et al. [10] obtained the stiffness and damping characteristics of the elastomeric diaphragm indirectly by comparing the experimental and theoretical results. Additionally, Pu established the stiffness and damping model using a similar approach in other research. Since the 1990s, analog simulation has become an attractive method for examining the pneumatic spring, and the stiffness characteristic is often studied using the finite element method (FEM) [11]. However, experimental modification or the FEM is inconvenient for the parameter optimization design of a pneumatic isolator. Significant error 


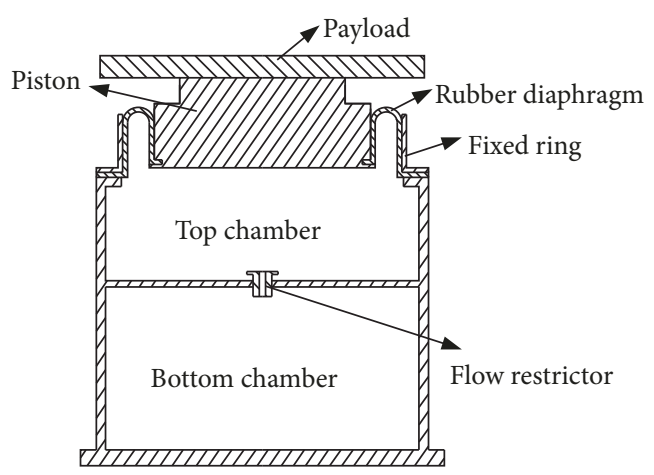

(a) Schematic of a pneumatic vibration isolator

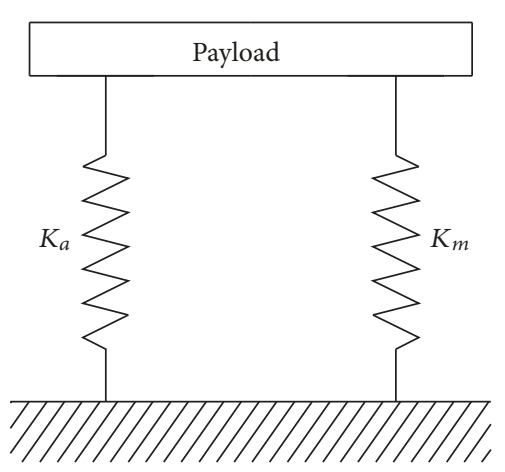

(b) Simplification of a pneumatic vibration isolator

Figure 1: Pneumatic vibration isolator.

typically still exists between the actual behavior and the presented model after the experimental modification or FEM study. Therefore, a direct theoretical derivation method must be established for the development of an analytical model for a pneumatic vibration isolator.

Zhu et al. [12] and Li [13] proposed an analytic model of an elastomeric belt in a magnetic-levitation vibration isolator using the Mooney-Rivlin model, and the model was validated experimentally. In that research, the elastomeric belt was chosen as the research object, and an accurate model was obtained according to direct theoretical derivation. Inspired by this work, we use the same method and theory to study and analyze the elastomeric diaphragm in pneumatic vibration absorbers and develop an analytical model of the elastomeric diaphragm in this paper. Specifically, because the elastomeric diaphragm is composed of several segments in the axial section, each segment can be simplified as uniaxial stretching after considering the structural restriction, and a force-strain equation can be established for each segment. The analytical model of the elastomeric diaphragm can be built by combining all segments, and the model can be resolved by numerical solution. Experimental tests were conducted, and the results demonstrate that the analytically predicted behavior is similar to the practically observed behavior. The error in this method can be reduced to less than $10 \%$ after the elastomeric diaphragm model is added to the standard pneumatic cylinder model. Although certain scholars have proposed several improved models that also imitate the practical behavior, this paper supplies a direct theoretical derivation method with a notably small error. The proposed analytical model facilitates the practical parameter optimization design of pneumatic isolators.

\section{Mathematic Model of the Elastomeric Diaphragm}

2.1. Configuration of the Pneumatic Vibration Isolator. The configuration of a typical pneumatic vibration isolator is shown in Figure 1(a), which includes an air-filled cylinder, elastomeric diaphragm, and piston. The cylinder consists of a top chamber and bottom chamber that are both filled with compressed air. The elastomeric diaphragm is located between the piston and cylinder, which is the seal component of the cylinder. The payload is connected to the piston, and the flow restrictor supplies damping for the vibration isolator.

Figure 1(b) illustrates that the payload is supported by two components: the pressurized air in the pneumatic cylinder and the elastomeric diaphragm, the model of which is often ignored. This paper only analyzes the stiffness effect of the elastomeric diaphragm without considering the damping effect because the vibration damping effect of the elastomeric diaphragm is a highly complex phenomenon. Therefore, the total stiffness of the pneumatic spring isolators can be obtained by adding the vertical stiffness of the elastomeric diaphragm (the model typically ignored in previous analysis and research) to the vertical stiffness of the pressurized air. The total stiffness of the pneumatic spring can be expressed as follows [14]:

$$
K_{\text {total }}=K_{m}+K_{a}
$$

where $K_{\text {total }}$ represents the total stiffness of the pneumatic spring isolators, $K_{a}$ represents the vertical stiffness of the compressed air, and $K_{m}$ represents the vertical stiffness of the elastomeric diaphragm. $K_{m}$ has typically been neglected in previous analyses of the pneumatic spring, which causes a certain error between the theory and experiments. This error in the total vertical stiffness can result in a series of problems. For example, the actual inherent frequency of a pneumatic vibration absorber cannot be easily estimated with high accuracy. After considering the effect of elastomeric diaphragm, a complete analytical model for a pneumatic vibration isolator is developed, and the developed analytical model can be used in the parameter optimization design of a pneumatic vibration isolator when the total stiffness and inherent frequency of the pneumatic vibration absorber are both known.

Figure 2 shows a cross-sectional view of an elastomeric diaphragm, and the parameters of the elastomeric diaphragm are listed in Table 1. After the elastomeric diaphragm is divided into four segments, the vertical stiffness of the segments, expressed as $K_{1}, K_{2}, K_{3}$, and $K_{4}$, is deduced such 
TABLE 1: Parameters of the elastomeric diaphragm.

\begin{tabular}{lc}
\hline Symbol & Description \\
\hline$R$ & Radius of the piston (radius of the inner circle of the elastomeric diaphragm) \\
$r$ & Radius of the arc of the elastomeric diaphragm \\
$h_{1}$ & Height of the inner circle of the elastomeric diaphragm \\
$h_{3}$ & Height of the outer circle \\
$d_{0}$ & Thickness of the elastomeric diaphragm \\
"1" & Inner circle of the elastomeric diaphragm \\
"2" & Inner arc of the elastomeric diaphragm \\
" 4 " & Outer circle of the elastomeric diaphragm \\
\hline
\end{tabular}

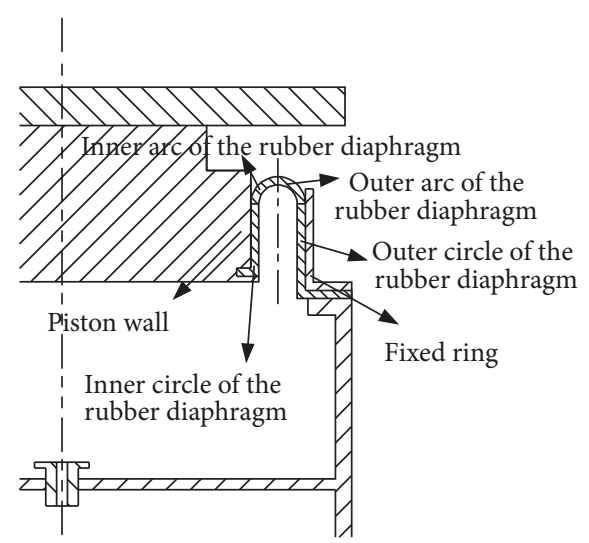

(a) Size definition of the elastomeric diaphragm

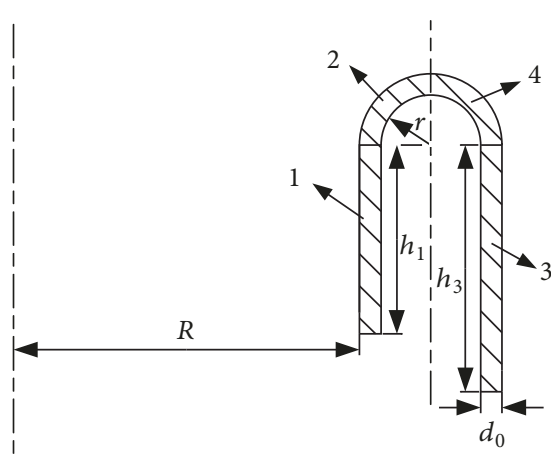

(b) Parameters of the elastomeric diaphragm

FIGURE 2: Elastomeric diaphragm.

that the total vertical stiffness of the elastomeric diaphragm can be determined.

2.2. Force Analysis of the Elastomeric Diaphragm. The forces of the piston and elastomeric diaphragm in the vertical direction are shown in Figure 3; then,

$$
\begin{aligned}
F_{a}+F_{1} & =G+F_{0} \\
F_{1} & =F_{1}^{\prime}=F_{3}=F_{3}^{\prime}=\frac{F_{P}}{2}
\end{aligned}
$$

where $F_{1}$ is the pulling force of part " 2 " acting on part " 1 " and $F_{3}$ is the pulling force of part " 4 " acting on part " 3 ". In addition, $F_{1}^{\prime}$ and $F_{3}^{\prime}$ are the reaction forces of $F_{1}$ and $F_{3}$, respectively, and $F_{a}, F_{0}$ and $G$ can be rewritten as

$$
\begin{aligned}
& F_{a}=P_{a} \cdot \pi\left(R+d_{0} \lambda_{31}\right)^{2} \\
& F_{0}=P_{0} \cdot \pi\left(R+d_{0} \lambda_{31}\right)^{2} \\
& G=m g
\end{aligned}
$$

where $F_{a}$ is the vertical force of the air in the chamber acting on the effective piston area, $F_{0}$ is the vertical force of the air outside the chamber acting on the effective piston area, $G$ is the gravity of the load, $P_{0}$ is the atmospheric pressure, $P_{a}$ is the air pressure in the chamber, $m$ is the payload mass, $g$ is the gravitational acceleration, and $\lambda_{31}$ is the corresponding stretch ratio of a unit of part "1", which is explained later. The stretch ratios in this paper are defined as the ratios of the stretched length to the unstretched length or original length of a small-volume element, which is a cube when it is unstretched or in the original state.

As shown in Figure 3(b), $F_{p}$ is the force acting on the arc of the elastomeric diaphragm in the vertical direction and is expressed as

$$
F_{P}=\left(P_{a}-P_{0}\right) \cdot s_{t}
$$

where $s_{t}$ is the projection area of the arc of the elastomeric diaphragm, as shown in Figure 4 . Then, $s_{t}$ can be expressed as

$$
s_{t}=\pi \cdot\left(R+2 d_{0}+2 \mathrm{r}-d_{0} \lambda_{33}\right)^{2}-\pi \cdot\left(d_{0} \lambda_{31}+R\right)^{2}
$$

where $\lambda_{33}$ is the corresponding stretch ratio of a unit of part " 3 ", which is explained later.

Substituting (8) into (7) leads to

$$
\begin{aligned}
F_{P}= & \pi\left(P_{a}-P_{0}\right) \\
& \cdot\left[\left(R+2 d_{0}+2 \mathrm{r}-d_{0} \lambda_{33}\right)^{2}-\left(d_{0} \lambda_{31}+R\right)^{2}\right] .
\end{aligned}
$$




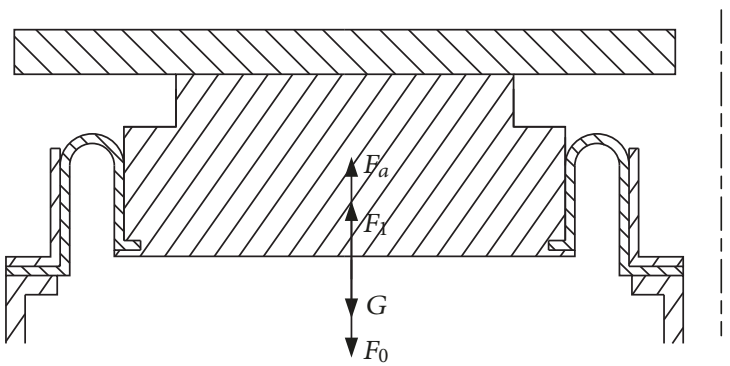

(a) Force analysis of the piston

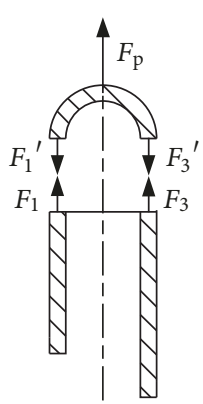

(b) Force analysis of the elastomeric diaphragm

FIGURE 3: Force analysis of the piston and elastomeric diaphragm.

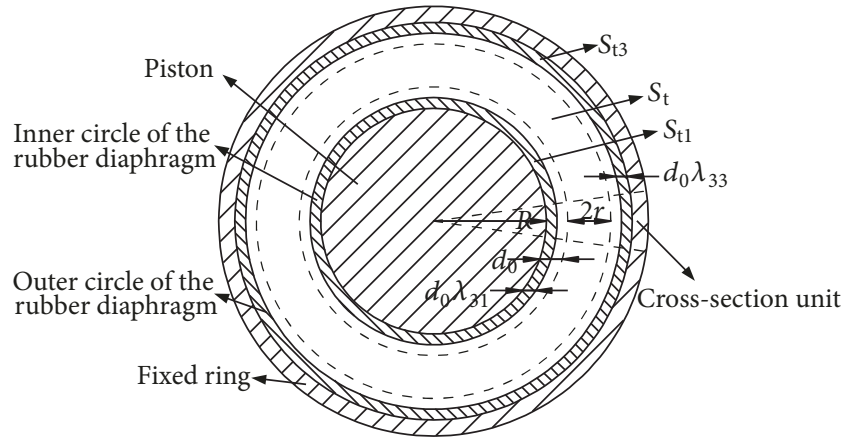

FIGURE 4: Cross-section of the dual-chamber pneumatic spring.

By substituting (9) into (3) and combining the result with (2), (4), (5), and (6), the air pressure in the chamber can be obtained as

$$
\begin{aligned}
P_{a}= & \frac{2 m g}{\pi\left[\left(R+2 d_{0}+2 \mathrm{r}-d_{0} \lambda_{33}\right)^{2}+\left(d_{0} \lambda_{31}+R\right)^{2}\right]} \\
& +P_{0} .
\end{aligned}
$$

By substituting (10) into (9) and noting (3), one can obtain

$$
\begin{aligned}
F_{1} & =F_{3}=\frac{F_{P}}{2} \\
& =\frac{m g\left[\left(R+2 d_{0}+2 \mathrm{r}-d_{0} \lambda_{33}\right)^{2}-\left(d_{0} \lambda_{31}+R\right)^{2}\right]}{\left(R+2 d_{0}+2 \mathrm{r}-d_{0} \lambda_{33}\right)^{2}+\left(d_{0} \lambda_{31}+R\right)^{2}} .
\end{aligned}
$$

2.3. Mooney-Rivlin Model. Mooney-Rivlin $[15,16]$ assumed that the elastic behavior was isotropic and that simple stretching satisfied Hooke's law; then, they obtained the rubber energy density function $W$ under the premise of not changing the rubber state and compressing its volume; i.e.,

$$
W=W\left(J_{1}, J_{2}, J_{3}\right)
$$

and

$$
\begin{aligned}
& J_{1}=\lambda_{1}^{2}+\lambda_{2}^{2}+\lambda_{3}^{2}-3 \\
& J_{2}=\left(\lambda_{1} \lambda_{2}\right)^{2}+\left(\lambda_{2} \lambda_{3}\right)^{2}+\left(\lambda_{1} \lambda_{3}\right)^{2}-3 \\
& J_{3}=\lambda_{1}^{2} \lambda_{2}^{2} \lambda_{3}^{2}-1
\end{aligned}
$$

where $\lambda_{1}, \lambda_{2}$, and $\lambda_{3}$ represent the stretch ratios in three different directions and are defined as the ratios of the stretched length to the unstretched length of a small-volume element, which is a cube when it is unstretched. These three directions, which are related to $\lambda_{1}, \lambda_{2}$, and $\lambda_{3}$, are expressed under generalized XYZ coordinates. If $\varepsilon_{i}$ is defined as a corresponding stretched length, the stretch ratio can be expressed as $\lambda_{i}=1+\varepsilon_{i} / 1$. Herein, the rubber satisfies the incompressibility condition, i.e., $\lambda_{1} \lambda_{2} \lambda_{3}=1$, which means that $J_{3}=0$. The corresponding deformation energy density $W$ only depends on $J_{1}$ and $J_{2}$. Under the condition of sufficiently small deformation, the form of deformation energy function can be expressed as

$$
\begin{aligned}
W & =C_{1} J_{1}+C_{2} J_{2} \\
C_{1} & =\frac{\partial W}{\partial J_{1}}, \\
C_{2} & =\frac{\partial W}{\partial J_{2}}
\end{aligned}
$$

where $C_{1}$ and $C_{2}$ represent different material parameters.

\subsection{Stiffness Analysis of the Elastomeric Diaphragm}

2.4.1. Part "1" Stiffness of the Elastomeric Diaphragm. As shown in Figure 5(a), when an elastomeric diaphragm is stretched, the tension $F_{1}$ acting on part "1" can be expressed as

$$
F_{1}=\delta_{11} s_{t 1}
$$

where $s_{t 1}$ is the sectional area of part "1", which is shown in Figure 4 , and $s_{t 1}$ can be expressed as

$$
s_{t 1}=\pi\left[\left(R+d_{0} \lambda_{31}\right)^{2}-R^{2}\right]
$$

where $\lambda_{31}$ is the corresponding stretch ratio of a unit of part " 1 ". In this paper, the letters of the subscript contain two numbers $i$ and $j$, where $i(1,2,3)$ represents the corresponding principal stress surface and $j(1,2,3,4)$ represents the corresponding component of the elastomeric diaphragm, i.e., the inner circle of the elastomeric diaphragm, the inner arc of 


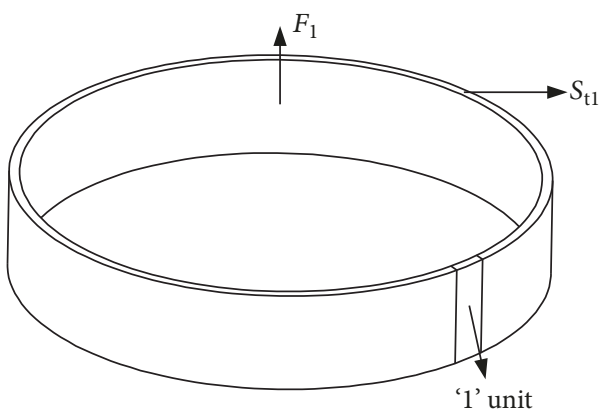

(a) Schematic diagram and force analysis of part " 1 "

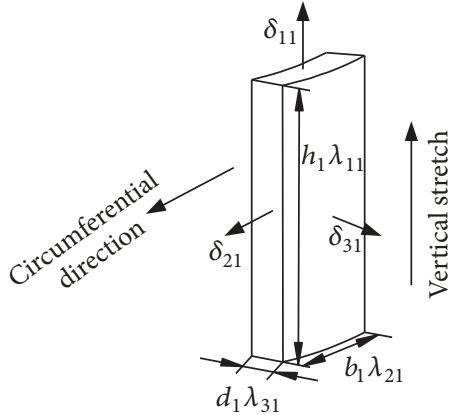

(b) Parameters and force analysis of "1" unit of part "1"

Figure 5: Force analysis of part "1" of the elastomeric diaphragm.

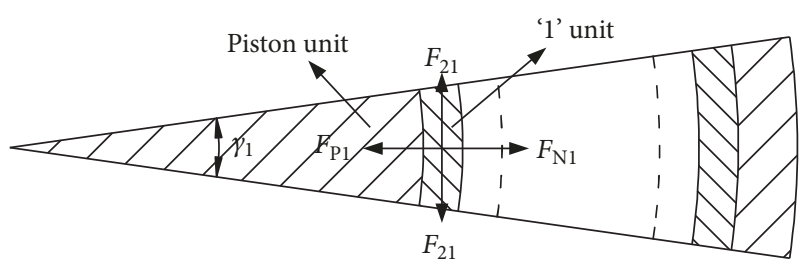

FIGURE 6: Cross-section of one unit of part "1".

the elastomeric diaphragm, the outer circle of the elastomeric diaphragm, and the outer arc of the elastomeric diaphragm, respectively.

Figure 5(b) shows the unit of part " 1 ", and the unit stress in three directions can be expressed as

$$
\begin{aligned}
& \delta_{11}=2\left[\mathrm{C}_{1} \lambda_{11}^{2}-\mathrm{C}_{2} \lambda_{11}^{-2}\right]+P_{1} \\
& \delta_{21}=2\left[\mathrm{C}_{1} \lambda_{21}^{2}-\mathrm{C}_{2} \lambda_{21}^{-2}\right]+P_{1} \\
& \delta_{31}=2\left[\mathrm{C}_{1} \lambda_{31}^{2}-C_{2} \lambda_{31}^{-2}\right]+P_{1}=P_{a}
\end{aligned}
$$

where $\lambda_{i j}$ is the corresponding stretch ratio and $P_{1}$ is the unknown pressure. The stretch ratio can be expressed as $\lambda_{11}=\left(h_{1}+\varepsilon_{1}\right) / h_{1}$, where $\varepsilon_{1}$ is the length variable in the vertical direction of part " 1 ".

The following equation can be obtained by combining (18) with (20):

$$
\delta_{11}=P_{a}+2\left[\mathrm{C}_{1} \lambda_{11}^{2}-\mathrm{C}_{2} \lambda_{11}^{-2}\right]-2\left[\mathrm{C}_{1} \lambda_{31}^{2}-\mathrm{C}_{2} \lambda_{31}^{-2}\right] .
$$

Substituting (17) and (21) into (16) yields

$$
\begin{aligned}
F_{1} & =\pi\left\{P_{a}+2\left[C_{1} \lambda_{11}^{2}-C_{2} \lambda_{11}^{-2}\right]\right. \\
& \left.-2\left[C_{1} \lambda_{31}^{2}-C_{2} \lambda_{31}^{-2}\right]\right\}\left[\left(R+d_{0} \lambda_{31}\right)^{2}-R^{2}\right] .
\end{aligned}
$$

The force on the unit in the cross-section is shown in Figure 6. The force of the piston acting on the unit of part " 1 " can be expressed as

$$
\mathrm{F}_{N 1}=F_{P 1}+2 F_{21} \cdot \sin \left(\frac{\gamma_{1}}{2}\right)
$$

where $F_{p 1}$ is the third principal stress surface of part " 1 " subjected to the force of the gas chamber, $F_{21}$ is the second principal stress surface of part " 1 " subjected to the force, and $\gamma_{1}$ is the angle of the unit in the cross-section of the dualchamber pneumatic spring.

$F_{P 1}$ and $F_{21}$ are defined as

$$
\begin{aligned}
& F_{P 1}=P_{a} \cdot h_{1} \lambda_{11} \cdot b_{1} \lambda_{21} \\
& F_{21}=\delta_{21} \cdot h_{1} \lambda_{11} \cdot d_{0} \lambda_{31}
\end{aligned}
$$

where $h_{1} \lambda_{11} \cdot b_{1} \lambda_{21}$ is the area of the third principal stress surface of part "1", $\delta_{21}$ is the stress of the second principal stress surface of part " 1 ", and $h_{1} \lambda_{11} \cdot d_{0} \lambda_{31}$ is the area of the second principal stress surface of part "1", as shown in Figure 5(b).

Substituting (24) and (25) into (23) yields

$$
\begin{aligned}
F_{N 1}= & P_{a} \cdot h_{1} \lambda_{11} \cdot b_{1} \lambda_{21}+2 \delta_{21} \cdot h_{1} \lambda_{11} \cdot d_{0} \lambda_{31} \\
& \cdot \sin \left(\frac{\gamma_{1}}{2}\right)
\end{aligned}
$$

which indicates that $F_{N 1}>0$. Therefore, part "1" of the elastomeric diaphragm remains in contact with the piston wall, and the size of part "1" of the elastomeric diaphragm does not change when it is stretched in the circumferential direction; i.e., $\lambda_{21}=1$. The incompressibility of rubber leads to $\lambda_{11} \lambda_{21} \lambda_{31}=1$, and the following expression can be obtained:

$$
\lambda_{31}=\lambda_{11}^{-1}=\left(\frac{h_{1}+\varepsilon_{1}}{h_{1}}\right)^{-1} .
$$

Substituting (10) and (27) into (22) yields 


$$
\begin{aligned}
F_{1} & =\pi\left\{\frac{2 m g}{\pi\left[\left(R+2 d_{0}+2 r-d_{0} h_{3} /\left(h_{3}+\varepsilon_{3}\right)\right)^{2}+\left(d_{0} h_{1} /\left(h_{1}+\varepsilon_{1}\right)+R\right)^{2}\right]}+P_{0}\right. \\
& \left.+2\left(C_{1}+C_{2}\right)\left[\left(\frac{h_{1}+\varepsilon_{1}}{h_{1}}\right)^{2}-\left(\frac{h_{1}}{h_{1}+\varepsilon_{1}}\right)^{2}\right]\right\}\left[\left(R+\frac{h_{1} d_{0}}{h_{1}+\varepsilon_{1}}\right)^{2}-R^{2}\right] .
\end{aligned}
$$

To obtain a function for the stiffness $K_{1}$ of part " 1 " with respect to variable $\varepsilon_{1}$, the following equation can be obtained by differentiating (28) with respect to $\varepsilon_{1}$ :

$$
K_{1}=\frac{d F_{1}}{d \varepsilon_{1}} .
$$

The complete equation (29) is extremely long and is provided in Appendix B.

2.4.2. Part "2" Stiffness of the Elastomeric Diaphragm. As shown in Figure 7, the tension $F_{2}$ acting on part " 2 " can be expressed as

$$
F_{2}=\int_{0}^{\pi / 2} \int_{0}^{2 \pi} \delta_{2} d s_{t 2} \cdot d \alpha
$$

where $F_{2}$ originates from the pressure difference of the top chamber and ambient environment in the vertical direction.

For the projected area of the unit, $d s_{t 2}$ can be written as

$$
d s_{t 2}=(R+r(1-\cos \alpha)) d \theta \cdot d_{0} \lambda_{32} \cdot \cos \alpha
$$

where $R+r(1-\cos \alpha)$ is the length $l_{2}$ from the analysis point to the center axis, $(R+r(1-\cos \alpha)) d \theta$ is the side length $d_{2}$ of the unit in part " 2 ", and $d_{0} \lambda_{32}$ is the thickness of the unit in part " 2 ". $\theta$ is shown in Figure 7(a).
The vertical stress $\delta_{2}$ of the unit of part " 2 " can be expressed as

$$
\delta_{2}=\delta_{12} \cos \alpha+\delta_{32} \sin \alpha
$$

where $\delta_{12}$ and $\delta_{32}$ can be written as

$$
\begin{aligned}
& \delta_{12}=2\left[C_{1} \lambda_{12}^{2}-C_{2} \lambda_{12}^{-2}\right]+P_{2} \\
& \delta_{32}=2\left[C_{1} \lambda_{32}^{2}-C_{2} \lambda_{32}^{-2}\right]+P_{2}=P_{a}-P_{0}
\end{aligned}
$$

where $\delta_{12}$ and $\delta_{32}$ are the stresses of the corresponding principal stress surface and $P_{2}$ is the unknown pressure. The stretch ratio can be expressed as $\lambda_{12}=\left(r+\varepsilon_{2}\right) / r$, where $\varepsilon_{2}$ is the length variable in the vertical direction. The size of part " 2 " does not change when it is stretched in the circumferential direction; i.e., $\lambda_{22}=1$. The incompressibility of rubber leads to $\lambda_{12} \lambda_{22} \lambda_{32}=1$. Thus, the following equation can be obtained:

$$
\lambda_{32}=\lambda_{12}^{-1}=\left(\frac{r+\varepsilon_{2}}{r}\right)^{-1}
$$

The following equation can be obtained by transferring (32) to (35):

$$
\begin{aligned}
\delta_{2} & =\left\{P_{a}-P_{0}\right. \\
& \left.+2\left(C_{1}+C_{2}\right)\left[\left(\frac{r+\varepsilon_{2}}{r}\right)^{2}-\left(\frac{r}{r+\varepsilon_{2}}\right)^{2}\right]\right\} \cos \alpha \\
& +\left(P_{a}-P_{0}\right) \sin \alpha .
\end{aligned}
$$

Substituting (10), (30), (31), and (36) into (30) yields

$$
\begin{aligned}
F_{2} & =2 \pi\left\{\left[\frac{2 m g}{\pi\left[\left(R+2 d_{0}+2 r-d_{0} h_{3} /\left(h_{3}+\varepsilon_{3}\right)\right)^{2}+\left(d_{0} h_{1} /\left(h_{1}+\varepsilon_{1}\right)+R\right)^{2}\right]}+2\left(C_{1}+C_{2}\right)\left(\left(\frac{r+\varepsilon_{2}}{r}\right)^{2}-\left(\frac{r}{r+\varepsilon_{2}}\right)^{2}\right)\right]\right. \\
& \cdot\left[\frac{\pi d_{0} r(R+r)}{4\left(r+\varepsilon_{2}\right)}-\frac{2 d_{0} r^{2}}{3\left(r+\varepsilon_{2}\right)}\right] \\
& \left.+\frac{2 m g}{\pi\left[\left(R+2 d_{0}+2 r-d_{0} h_{3} /\left(h_{3}+\varepsilon_{3}\right)\right)^{2}+\left(d_{0} h_{1} /\left(h_{1}+\varepsilon_{1}\right)+R\right)^{2}\right]}\left[\left(\frac{d_{0} r(R+r)}{\left(r+\varepsilon_{2}\right)}\right)-\frac{d_{0} r^{2}}{3\left(r+\varepsilon_{2}\right)}\right]\right\}
\end{aligned}
$$




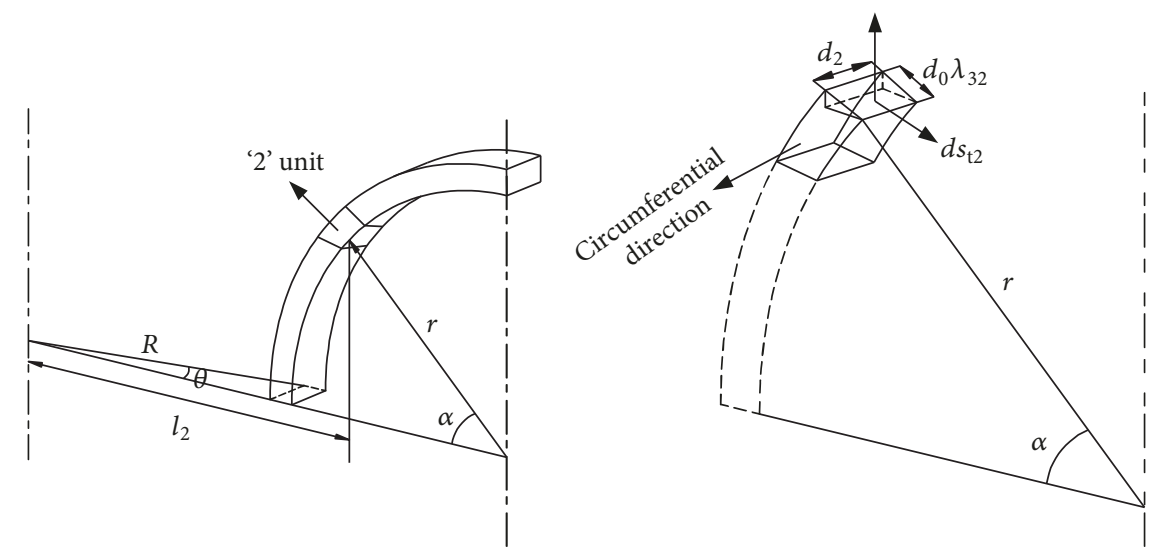

(a) Schematic diagram of part "2"

(b) Parameters of "2" unit of part "2"

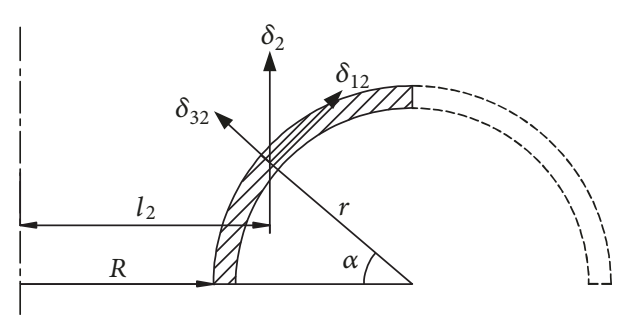

(c) Force analysis of "2" unit of part "2"

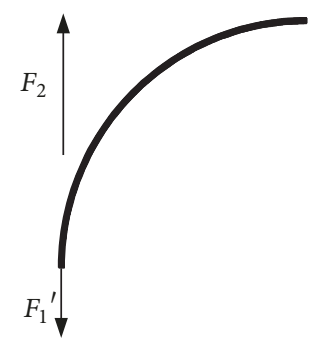

(d) Force analysis of part

FIGURE 7: Force analysis of part "2" of the elastomeric diaphragm.

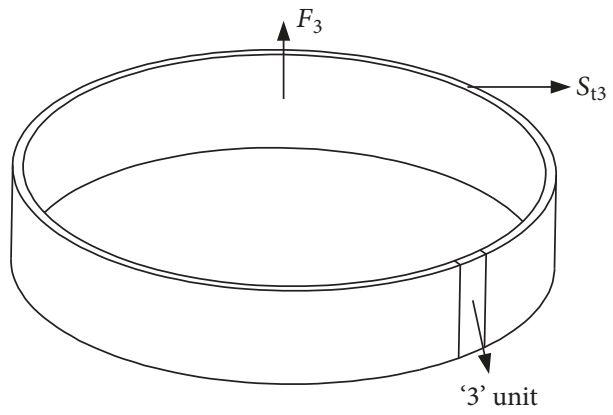

(a) Schematic diagram and force analysis of part " 3 "

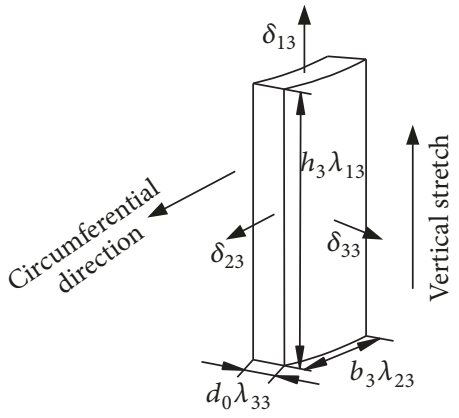

(b) Parameters and force analysis of " 3 " unit of part " 3 "

FIGURE 8: Force analysis of part " 3 " of the elastomeric diaphragm.

To obtain a function of the stiffness $K_{2}$ of part "2" with respect to variable $\varepsilon_{2}$, the following equation can be obtained by differentiating (37) with respect to $\varepsilon_{2}$ :

$$
K_{2}=\frac{d F_{2}}{d \varepsilon_{2}} .
$$

The complete equation (38) is extremely long and is provided in Appendix B.

2.4.3. Part "3" Stiffness of the Elastomeric Diaphragm. As shown in Figure 8, when an elastomeric diaphragm is stretched, the tension $F_{3}$ acting on part " 3 " can be expressed as

$$
F_{3}=\delta_{13} s_{t 3}
$$

The analysis of part " 3 " is similar to that of part " 1 ". Therefore, based on the Mooney-Rivlin model, the vertical stress $\delta_{13}$ can be expressed as

$$
\delta_{13}=P_{a}+2\left[\mathrm{C}_{1} \lambda_{13}^{2}-C_{2} \lambda_{13}^{-2}\right]-2\left[\mathrm{C}_{1} \lambda_{33}^{2}-C_{2} \lambda_{33}^{-2}\right] .
$$

where the stretch ratio can be expressed as $\lambda_{13}=\left(h_{3}+\varepsilon_{3}\right) / h_{3}$ and $\varepsilon_{3}$ is the variable length in the vertical stretch of part " 3 ". 


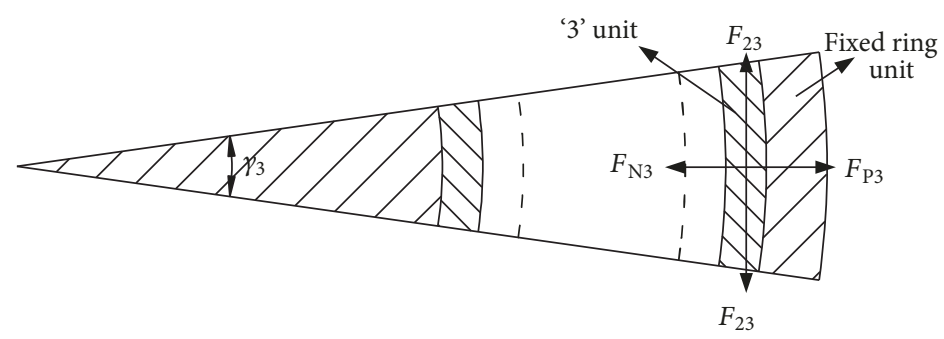

FIGURE 9: Cross-section of one unit of part “3”.

Additionally, $s_{t 3}$ is the cross-sectional area of part " 3 ", as shown in Figure 4 . Herein, $s_{t 3}$ can be expressed as

$$
s_{t 3}=\pi\left[\left(R+2 d_{0}+2 r\right)^{2}-\left(R+2 d_{0}+2 r-d_{0} \lambda_{33}\right)^{2}\right] .
$$

Substituting (40) and (41) into (39) yields

$$
\begin{aligned}
F_{3} & \pi\left\{P_{a}+2\left[C_{1} \lambda_{13}^{2}-C_{2} \lambda_{13}^{-2}\right]-2\left[C_{1} \lambda_{33}^{2}-C_{2} \lambda_{33}^{-2}\right]\right\} \\
& \cdot\left\{\left(R+2 d_{0}+2 r\right)^{2}-\left(R+2 d_{0}+2 r-d_{0} \lambda_{33}\right)^{2}\right\} .
\end{aligned}
$$

The force on the unit in the cross-section is shown in Figure 9. The force of the piston acting on the unit of part " 3 " can be expressed as

$$
F_{N 3}=F_{P 3}-2 F_{23} \cdot \sin \left(\frac{\gamma_{3}}{2}\right)
$$

where $F_{p 3}$ is the third principal stress surface of part " 3 " subjected to the force of the gas chamber, $F_{23}$ is the second principal stress surface of part " 3 " subjected to the force, and $\gamma_{3}$ is the angle of the unit in cross-section of the dual-chamber pneumatic spring.

$F_{P 3}$ and $F_{23}$ are written as

$$
\begin{aligned}
& F_{P 3}=P_{a} \cdot h_{3} \lambda_{13} \cdot b_{3} \lambda_{23} \\
& F_{23}=\delta_{23} \cdot h_{3} \lambda_{13} \cdot d_{0} \lambda_{33}
\end{aligned}
$$

where $h_{3} \lambda_{13} \cdot b_{3} \lambda_{23}$ is the area of the third principal stress surface of part " 3 ", $\delta_{23}$ is the stress of the second principal stress surface of part " 3 ", and $h_{3} \lambda_{13} \cdot d_{0} \lambda_{33}$ is the area of the second principal stress surface of part " 3 ", as shown in Figure 8(b).

Substituting (44) and (45) into (43) yields

$$
\begin{aligned}
F_{N 3}= & P_{a} \cdot h_{3} \lambda_{13} \cdot b_{3} \lambda_{23}-2 \delta_{23} \cdot h_{3} \lambda_{13} \cdot d_{0} \lambda_{33} \\
& \cdot \sin \left(\frac{\gamma_{3}}{2}\right)
\end{aligned}
$$

where $\sin \left(\gamma_{3} / 2\right)=b_{3} / 2\left(R+2 r+2 d_{0}-d_{0} \lambda_{33}\right)$ and $b_{3}$ is the arc length of the unit of part " 3 ", which is smaller than $2\left(R+2 r+2 d_{0}-d_{0} \lambda_{33}\right)$. Thus, the first polynomial is larger than the second polynomial of (46); i.e., $F_{N 3}>0$. Therefore, part " 3 " remains in contact with the fixed ring, and the size of part " 3 " does not change when it is stretched in the circumferential direction; i.e., $\lambda_{23}=1$. However, the incompressibility of rubber leads to $\lambda_{13} \lambda_{23} \lambda_{33}=1$. Thus, the following equation can be obtained:

$$
\lambda_{33}=\lambda_{13}^{-1}=\left(\frac{h_{3}+\varepsilon_{3}}{h_{3}}\right)^{-1} .
$$

Substituting (10) and (47) into (42) yields

$$
\begin{aligned}
F_{3} & =\pi\left\{\frac{2 m g}{\pi\left[\left(R+2 d_{0}+2 r-d_{0} h_{3} /\left(h_{3}+\varepsilon_{3}\right)\right)^{2}+\left(d_{0} h_{1} /\left(h_{1}+\varepsilon_{1}\right)+R\right)^{2}\right]}+P_{0}\right. \\
& \left.+2\left(C_{1}+C_{2}\right)\left[\left(\frac{h_{3}+\varepsilon_{3}}{h_{3}}\right)^{2}-\left(\frac{h_{3}}{h_{3}+\varepsilon_{3}}\right)^{2}\right]\right\}\left[\left(R+2 d_{0}+2 r\right)^{2}-\left(R+2 d_{0}+2 r-\frac{d_{0} h_{3}}{h_{3}+\varepsilon_{3}}\right)^{2}\right] .
\end{aligned}
$$

To obtain a function of the stiffness $K_{3}$ of part " 3 " with respect to variable $\varepsilon_{3}$, the following equation can be obtained by differentiating (48) with respect to $\varepsilon_{3}$ :

$$
K_{3}=\frac{d F_{3}}{d \varepsilon_{3}} .
$$

The complete equation (49) is extremely long and is provided in Appendix B.

2.4.4. Part "4" Stiffness of the Elastomeric Diaphragm. As shown in Figure 10, the tension $F_{4}$ acting on part " 4 " can be expressed as 


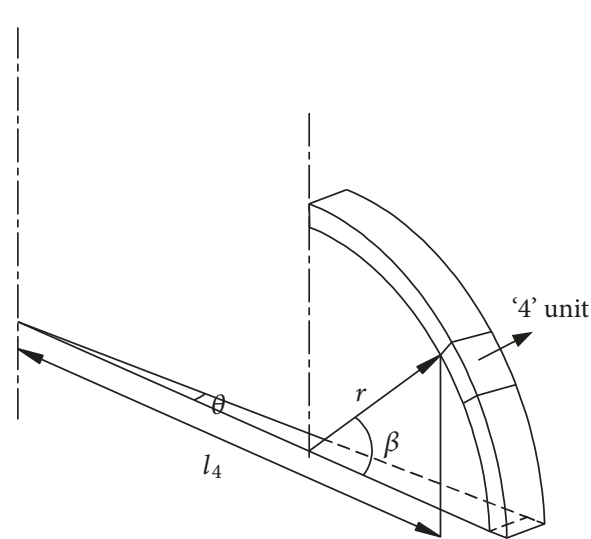

(a) Schematic diagram of part " 4 "

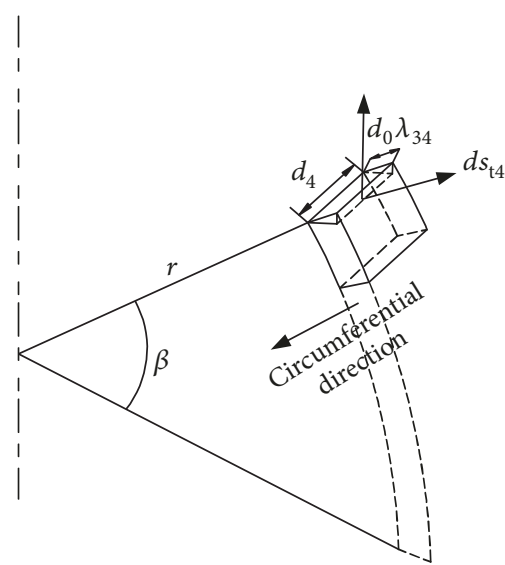

(b) Parameters of the " 4 " unit of part " 4 "

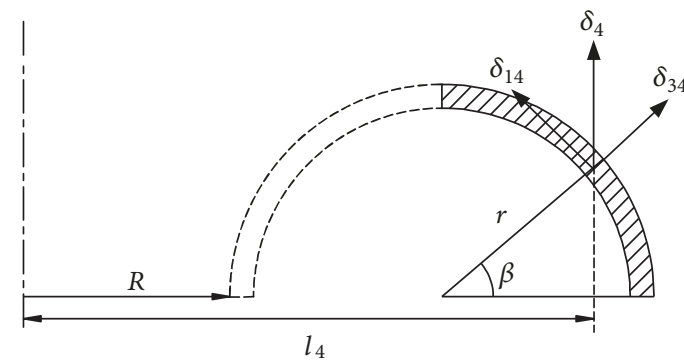

(c) Force analysis of the " 4 " unit of part " 4 "

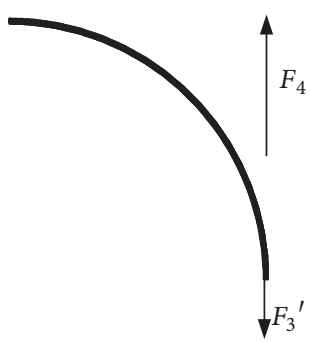

(d) Force analysis of part

Figure 10: Force analysis of part "4" of the elastomeric diaphragm.

$$
F_{4}=\int_{0}^{\pi / 2} \int_{0}^{2 \pi} \delta_{4} d s_{t 4} \cdot d \beta
$$

where $F_{4}$ originates from the pressure difference of the top chamber and the ambient environment in the vertical direction.

For the projected area of the unit, $d s_{t 4}$ can be written as

$$
d s_{t 4}=(R+r(1+\cos \beta)) d \theta \cdot d_{0} \lambda_{34} \cdot \cos \beta
$$

where $R+r(1+\cos \beta)$ is the length $l_{4}$ from the analysis point to the center axis, $(R+r(1+\cos \beta)) d \theta$ is the side length $d_{4}$ of the unit in part " 4 ", $d_{0} \lambda_{34}$ is the thickness of the unit in part " 4 ", and $\theta$ is shown in Figure 10(a).

The vertical stress $\delta_{4}$ of the partial unit in part " 4 " can be expressed as

$$
\delta_{4}=\delta_{14} \cos \beta+\delta_{34} \sin \beta
$$

and $\delta_{14}$ and $\delta_{34}$ can be rewritten as

$$
\begin{aligned}
& \delta_{14}=2\left[C_{1} \lambda_{14}^{2}-C_{2} \lambda_{14}^{-2}\right]+P_{4} \\
& \delta_{34}=2\left[C_{1} \lambda_{34}^{2}-C_{2} \lambda_{34}^{-2}\right]+P_{4}=P_{a}-P_{0}
\end{aligned}
$$

where $\delta_{14}$ and $\delta_{34}$ are the stresses corresponding to the principal stress surface and $P_{4}$ is the unknown pressure. The stretch ratio can be expressed as $\lambda_{14}=\left(r+\varepsilon_{4}\right) / r$, where $\varepsilon_{4}$ is the variable length in the vertical stretch of part " 4 ". The size of part " 4 " does not change when it is stretched in the circumferential direction; i.e., $\lambda_{24}=1$. The incompressibility of rubber leads to $\lambda_{14} \lambda_{24} \lambda_{34}=1$. Thus, the following equation can be obtained:

$$
\lambda_{34}=\lambda_{14}^{-1}=\left(\frac{r+\varepsilon_{4}}{r}\right)^{-1}
$$

The following equation can be obtained by transferring (52) to (55):

$$
\begin{aligned}
\delta_{4} & =\left\{P_{a}-P_{0}\right. \\
& \left.+2\left(C_{1}+C_{2}\right)\left[\left(\frac{r+\varepsilon_{4}}{r}\right)^{2}-\left(\frac{r+\varepsilon_{4}}{r}\right)^{-2}\right]\right\} \cos \beta \\
& +\left(P_{a}-P_{0}\right) \sin \beta .
\end{aligned}
$$

$$
F_{4}=2 \pi\left\{\left[\frac{2 m g}{\pi\left[\left(R+2 d_{0}+2 r-d_{0} h_{3} /\left(h_{3}+\varepsilon_{3}\right)\right)^{2}+\left(d_{0} h_{1} /\left(h_{1}+\varepsilon_{1}\right)+R\right)^{2}\right]}+2\left(C_{1}+C_{2}\right)\left(\left(\frac{r+\varepsilon_{4}}{r}\right)^{2}-\left(\frac{r}{r+\varepsilon_{4}}\right)^{2}\right)\right]\right.
$$




$$
\begin{aligned}
& \cdot\left[\frac{\pi d_{0} r(R+r)}{4\left(r+\varepsilon_{4}\right)}+\frac{2 d_{0} r^{2}}{3\left(r+\varepsilon_{4}\right)}\right] \\
& \left.+\frac{2 m g}{\pi\left[\left(R+2 d_{0}+2 r-d_{0} h_{3} /\left(h_{3}+\varepsilon_{3}\right)\right)^{2}+\left(d_{0} h_{1} /\left(h_{1}+\varepsilon_{1}\right)+R\right)^{2}\right]}\left[\frac{1}{2} d_{0}\left(\frac{r+\varepsilon_{4}}{r}\right)^{-1}(R+r)+\frac{1}{3} d_{0}\left(\frac{r+\varepsilon_{4}}{r}\right)^{-1} r\right]\right\} .
\end{aligned}
$$

To obtain a function of the stiffness $K_{4}$ of part " 4 " with respect to variable $\varepsilon_{4}$, the following equation can be obtained by differentiating (57) with respect to $\varepsilon_{4}$ :

$$
K_{4}=\frac{d F_{4}}{d \varepsilon_{4}}
$$

The entire equation (58) is extremely long and is provided in Appendix B.

2.4.5. Total Stiffness of the Elastomeric Diaphragm. As shown in Figure 11, part "1" and part " 2 " are connected in series such that the equivalent stiffness $K_{12}$ can be expressed as

$$
\frac{1}{K_{12}}=\frac{1}{K_{1}}+\frac{1}{K_{2}}
$$

Part " 3 " and part " 4 " are connected in series such that the equivalent stiffness $K_{34}$ can be expressed as

$$
\frac{1}{K_{34}}=\frac{1}{K_{3}}+\frac{1}{K_{4}}
$$

The total stiffness can be expressed as

$$
K_{m}=K_{12}+K_{34}=\frac{K_{1} K_{2}}{K_{1}+K_{2}}+\frac{K_{3} K_{4}}{K_{3}+K_{4}} .
$$

2.5. Numerical Solution of the Vertical Stiffness for the Elastomeric Diaphragm. When the load of the dual-chamber pneumatic spring is $m$, each part of the elastomeric diaphragm is stretched to a certain length. The stretched lengths of each corresponding part are defined as $\varepsilon_{10}, \varepsilon_{20}, \varepsilon_{30}$, and $\varepsilon_{40}$. By analyzing (3) and (11), each component of the force can be expressed as

$$
F_{1}=F_{1}^{\prime}=F_{2}=F_{3}=F_{3}^{\prime}=F_{4}=\frac{F_{P}}{2}=\frac{m g\left[\left(R+2 d_{0}+2 r-d_{0} h_{3} /\left(h_{3}+\varepsilon_{3}\right)\right)^{2}-\left(d_{0} h_{1} /\left(h_{1}+\varepsilon_{1}\right)+R\right)^{2}\right]}{\left(R+2 d_{0}+2 r-d_{0} h_{3} /\left(h_{3}+\varepsilon_{3}\right)\right)^{2}+\left(d_{0} h_{1} /\left(h_{1}+\varepsilon_{1}\right)+R\right)^{2}} .
$$

The analysis process to obtain the stretched length of each corresponding part with respect to the other variables is as follows. Substituting $\varepsilon_{1}=\varepsilon_{10}$ into (28) and noting (62), we can obtain (B.5) of the stretched length $\varepsilon_{10}$. (B.6), (B.7), and (B.8) can also be obtained in a similar manner. Equations (B.5) to (B.8) are rather long and are provided in Appendix B.

By solving (B.5) to (B.8) in the numerical root of $\varepsilon_{10}$, $\varepsilon_{20}, \varepsilon_{30}$, and $\varepsilon_{40}$ and substituting $\varepsilon_{1}=\varepsilon_{10}, \varepsilon_{2}=\varepsilon_{20}, \varepsilon_{3}=\varepsilon_{30}$, and $\varepsilon_{4}=\varepsilon_{40}$ into (29), (38), (49), and (58), we obtain the numerical solutions of stiffness $K_{1}, K_{2}, K_{3}$, and $K_{4}$, respectively. As a result, the total stiffness $K_{m}$ can be obtained from (61).

\section{Experimental Validation}

To verify the theoretical analysis, we test the performance and calculate the stiffness of the specific dual-chamber pneumatic spring.

The following text describes the method for obtaining $2\left(C_{1}+C_{2}\right)$, where $C_{1}$ and $C_{2}$ are the Mooney-Rivlin material parameters. Classical rubber theory yields the relationship between the stiffness values of different types of rubbers and their Young's elastic moduli [15]. The elastomeric diaphragms commonly used in design of the pneumatic spring structure consist of chloroprene rubber, nitrile butadiene rubber, butyl rubber, natural rubber, and other materials. A moderate stiffness is typically used to obtain a better overall performance. Therefore, the widely applied nitrile butadiene rubber with hardness $(55 \pm 5) H_{A}$ is used in the next two experiments. The Young's elastic modulus $E$ changes from 2.465 MPa to 3.619 $\mathrm{MPa}$ via the relationship between the hardness and Young's elastic modulus. According to the incompressibility of the rubber material, we can obtain $E \approx 3 G s$. Hence, $G s$ ranges from $0.82 \mathrm{MPa}$ to $1.20 \mathrm{MPa}$, where $G s$ is the shear modulus and $G s=2\left(C_{1}+C_{2}\right)$. Finally, $2\left(C_{1}+C_{2}\right)$ also ranges from 0.82 $\mathrm{MPa}$ to $1.20 \mathrm{MPa}$.

The vertical total stiffness $K_{\text {total }}$ of the dual-chamber pneumatic isolator can be calculated according to the parameters in Table 2, where the stiffness of the gas is obtained based on Erin's study. Two experiments are shown in Figures 12 and 13. The main components of the isolation platforms are composed of four dual-chamber pneumatic isolators. The microvibration of the ground can be attenuated by the dualchamber pneumatic isolators. Thus, we can obtain the transmissibility curve using the testing instrument HANDYSAM [17]. The input and output sensors used in this study are the ultralow frequency vibration pickup sensor 991B produced 
TABLE 2: Parameters of the dual-chamber pneumatic in the two experiments.

\begin{tabular}{|c|c|c|c|c|}
\hline Symbol & Description & Value 1 & Value 2 & Unit \\
\hline $2\left(C_{1}+C_{2}\right)$ & $C_{1}$ and $C_{2}$ are the Mooney-Rivlin material parameters & $0.82-1.20$ & $0.82-1.20$ & $\mathrm{MPa}$ \\
\hline$h_{1}$ & Part “1” vertical length & 0.009 & 0.015 & $\mathrm{~m}$ \\
\hline$r$ & Part "2" or " 4 " projection length & 0.0034 & 0.0050 & $\mathrm{~m}$ \\
\hline$h_{3}$ & Part “ 3 ” vertical length & 0.014 & 0.020 & $\mathrm{~m}$ \\
\hline$d_{0}$ & Diaphragm thickness & 0.0006 & 0.002 & $\mathrm{~m}$ \\
\hline$p_{0}$ & Atmospheric pressure & 101325 & 101325 & $\mathrm{MPa}$ \\
\hline$g$ & Gravitational acceleration & 9.81 & 9.81 & $\mathrm{~m} / \mathrm{s}^{2}$ \\
\hline$M$ & Payload mass & 20 & 375 & $\mathrm{~kg}$ \\
\hline$n$ & Polytropic exponent & 1.4 & 1.4 & - \\
\hline$L$ & Flow restrictor length & $4 e-4$ & $8 e-3$ & $\mathrm{~m}$ \\
\hline$D$ & Flow restrictor diameter & le-3 & $1 e-3$ & $\mathrm{~m}$ \\
\hline$A_{b}$ & Effective piston area & 0.0015 & 0.016 & $\mathrm{~m}^{2}$ \\
\hline$\mu$ & Air viscosity & $1.824 \mathrm{e}-5$ & $1.824 \mathrm{e}-5$ & $\mathrm{~Pa} \cdot \mathrm{s}$ \\
\hline$C_{r}$ & Flow restriction constant & $3.3640 \mathrm{e}-06$ & $1.6019 \mathrm{e}-07$ & $\mathrm{~m}^{3} /(\mathrm{s} \cdot \mathrm{Pa})$ \\
\hline$V_{b}$ & Lower chamber volume & $3.0000 \mathrm{e}-04$ & $1.8170 \mathrm{e}-04$ & $\mathrm{~m}^{3}$ \\
\hline$V_{t}$ & Upper chamber volume & $2.7298 \mathrm{e}-05$ & $2.3000 \mathrm{e}-03$ & $\mathrm{~m}^{3}$ \\
\hline
\end{tabular}

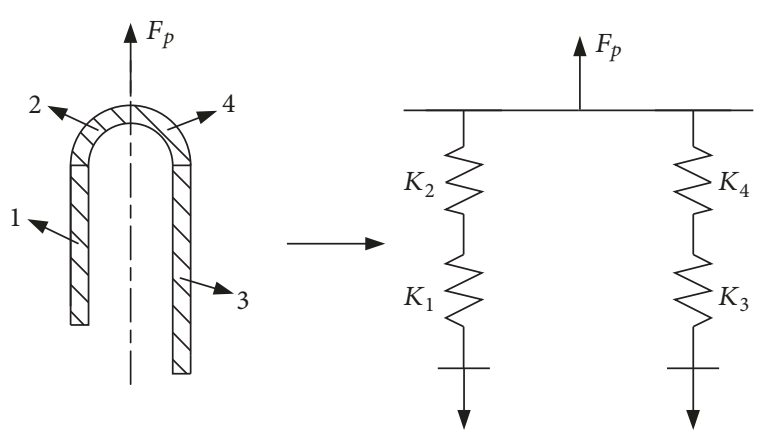

FIgURE 11: Total stiffness of the elastomeric diaphragm.

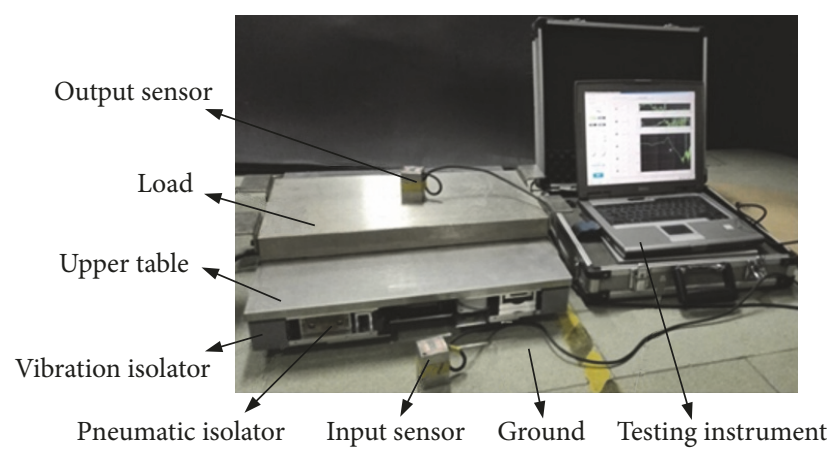

FIgURE 12: Photograph of experiment 1.

by the Institute of Engineering Mechanics, China Earthquake Administration. The sensitivity of the sensors is $4 \times 10^{-8} \mathrm{~m} / \mathrm{s}$. The load in experiment 1 is $50 \mathrm{~kg}$, and the load in experiment 2 is $200 \mathrm{~kg}$. Ground excitation used in the two experiments is just ambient vibration, whose signal in vertical direction measured by the input sensors is shown in Figure 14.

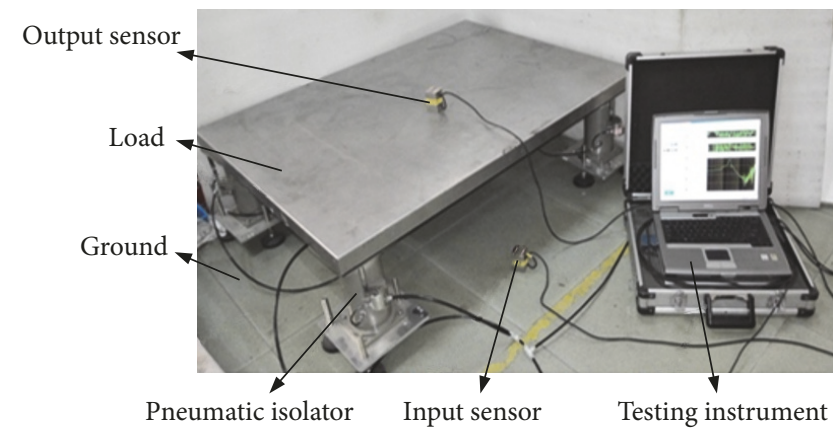

Figure 13: Photograph of experiment 2.

The theoretical expression of the frequency response functions has been inferred without considering the elastomeric diaphragm [10]. In this paper, the theoretical transmissibility curves are obtained as shown in Figures 15 and 16 by substituting the actual parameters of the experimental pneumatic vibration absorber shown in Table 2 into Erin's analytical model. The first curve is Erin's result without consideration of the elastomeric diaphragm. The second and third curves are our results with consideration of the elastomeric diaphragm. The last curve is the experimental result with the parameters shown in Table 2. Our model yields two curves, namely, the upper and lower bounds for the inherent frequencies, which correspond to the maximum and minimum values of $2\left(C_{1}+C_{2}\right)$.

The results of experiment 1 are shown in Figure 15, where $f_{a 1}=1.89 \mathrm{~Hz}, f_{C 1}=6.03-7.70 \mathrm{~Hz}$, and $f_{E 1}=6.75 \mathrm{~Hz}$ are the inherent frequencies of the double-chamber pneumatic springs based on Erin's study and the proposed theory in this paper that considers the elastomeric diaphragm stiffness and experiment results, respectively. The results of experiment 2 are shown in Figure 16, where $f_{a 2}=1.31 \mathrm{~Hz}, f_{\mathrm{C} 2}=3.38-4.30$ $\mathrm{Hz}$, and $f_{E 2}=3.75 \mathrm{~Hz}$ are the inherent frequencies based on 
TABLE 3: Stiffnesses from the calculations and experiments.

\begin{tabular}{|c|c|c|c|c|}
\hline Symbol & Description & Value 1 & Value 2 & Unit \\
\hline$\varepsilon_{10}$ & Part "1" length stretched & $15.0 \mathrm{e}-04$ to $9.19 \mathrm{e}-04$ & $22.0 \mathrm{e}-04$ to $14.0 \mathrm{e}-04$ & $\mathrm{~m}$ \\
\hline$\varepsilon_{20}$ & Part "2" length stretched & $8.53 \mathrm{e}-04$ to $5.16 \mathrm{e}-04$ & $9.90 \mathrm{e}-04$ to $5.85 \mathrm{e}-04$ & $\mathrm{~m}$ \\
\hline$\varepsilon_{30}$ & Part " 3 " length stretched & $13.0 \mathrm{e}-04$ to $8.55 \mathrm{e}-04$ & $18.0 \mathrm{e}-04$ to $11.0 \mathrm{e}-04$ & $\mathrm{~m}$ \\
\hline$\varepsilon_{40}$ & Part "4" length stretched & $6.26 \mathrm{e}-04$ to $3.88 \mathrm{e}-04$ & $8.23 \mathrm{e}-04$ to $4.94 \mathrm{e}-04$ & $\mathrm{~m}$ \\
\hline$K_{1}$ & Part “1” stiffness & $1.87 \mathrm{e}+04$ to $3.24 \mathrm{e}+04$ & $9.97 \mathrm{e}+04$ to $1.75 \mathrm{e}+05$ & $\mathrm{~N} / \mathrm{m}$ \\
\hline$K_{2}$ & Part "2" stiffness & $3.54 \mathrm{e}+04$ to $6.32 \mathrm{e}+04$ & $2.34 \mathrm{e}+05$ to $4.12 \mathrm{e}+05$ & $\mathrm{~N} / \mathrm{m}$ \\
\hline$K_{3}$ & Part “ 3 " stiffness & $1.90 \mathrm{e}+04$ to $3.10 \mathrm{e}+04$ & $1.04 \mathrm{e}+05$ to $1.73 \mathrm{e}+05$ & $\mathrm{~N} / \mathrm{m}$ \\
\hline$K_{4}$ & Part “4” stiffness & $4.84 \mathrm{e}+04$ to $8.42 \mathrm{e}+04$ & $2.52 \mathrm{e}+05$ to $4.54 \mathrm{e}+05$ & $\mathrm{~N} / \mathrm{m}$ \\
\hline$K_{12}$ & Part " 1 " and " 2 " equivalent stiffness & $1.23 \mathrm{e}+04$ to $2.14 \mathrm{e}+04$ & $6.99 \mathrm{e}+04$ to $1.23 \mathrm{e}+05$ & $\mathrm{~N} / \mathrm{m}$ \\
\hline$K_{34}$ & Part " 3 " and " 4 " equivalent stiffness & $1.36 \mathrm{e}+04$ to $2.27 \mathrm{e}+04$ & $7.35 \mathrm{e}+04$ to $1.25 \mathrm{e}+05$ & $\mathrm{~N} / \mathrm{m}$ \\
\hline$K_{m}$ & Rubber diaphragm stiffness & $2.59 \mathrm{e}+04$ to $4.41 \mathrm{e}+04$ & $1.43 \mathrm{e}+05$ to $2.48 \mathrm{e}+05$ & $\mathrm{~N} / \mathrm{m}$ \\
\hline$K_{a}$ & Air stiffness & $0.2850 \mathrm{e}+04$ & $2.5406 \mathrm{e}+04$ & $\mathrm{~N} / \mathrm{m}$ \\
\hline$K_{\text {total }}$ & Total stiffness & $2.87 \mathrm{e}+04$ to $4.90 \mathrm{e}+04$ & $1.69 \mathrm{e}+05$ to $2.74 \mathrm{e}+05$ & $\mathrm{~N} / \mathrm{m}$ \\
\hline$K_{E}$ & Experimental stiffness & $3.5975 \mathrm{e}+04$ & $2.0819 \mathrm{e}+05$ & $\mathrm{~N} / \mathrm{m}$ \\
\hline$f_{C}$ & Calculated inherent frequency & 6.0332 to 7.7096 & 3.3769 to 4.2931 & $\mathrm{~Hz}$ \\
\hline$f_{E}$ & Experimental inherent frequency & 6.625 & 3.5 & $\mathrm{~Hz}$ \\
\hline
\end{tabular}

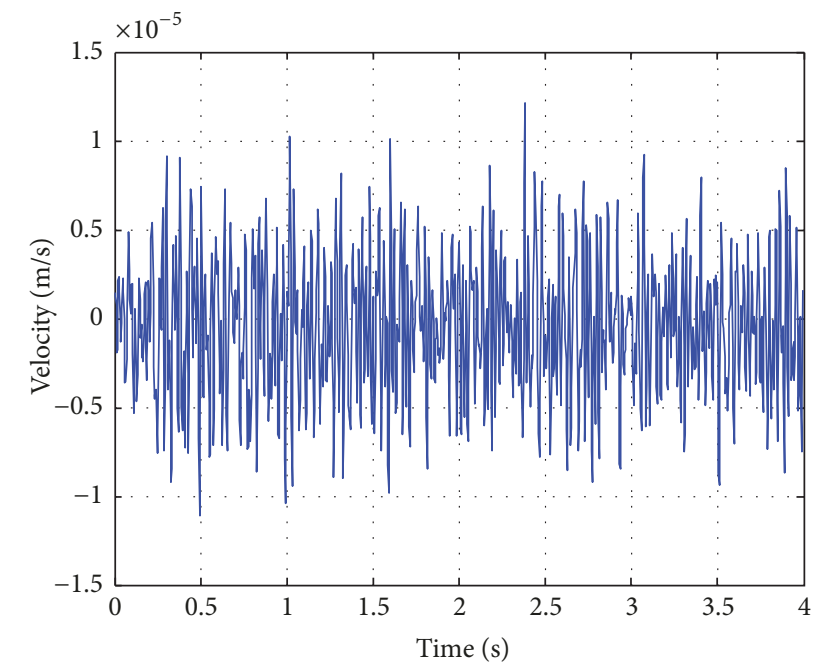

FIGURE 14: Ground vibration velocity curve in vertical direction versus time.

Erin's study and the proposed theoretical and experiment results, respectively. The value of the damping in our two experiments is related to both the length and diameter of the flow restrictor. In experiment 1 , the flow restrictor length is 0.0004 , and the flow restrictor diameter is 0.001 ; thus, the value of the damping ratio is 0.005 . In experiment 2 , the flow restrictor length is 0.008 , and the flow restrictor diameter is 0.016 ; thus, the value of the damping ratio is 0.1 .

Figures 15 and 16 illustrate that $f_{E 1}$ and $f_{E 2}$ are within the ranges of $f_{C 1}$ and $f_{C 2}$, respectively, demonstrating the effectiveness of the vertical stiffness model for the elastomeric diaphragm.

The stiffness values of the experiments can be obtained from the relationship of the inherent frequency, the stiffness, and the payload; i.e.,

$$
\begin{aligned}
& K_{E 1}=m \omega_{E 1}^{2}=m\left(2 \pi f_{E 1}\right)^{2}, \\
& K_{E 2}=m \omega_{E 2}^{2}=m\left(2 \pi f_{E 2}\right)^{2} .
\end{aligned}
$$

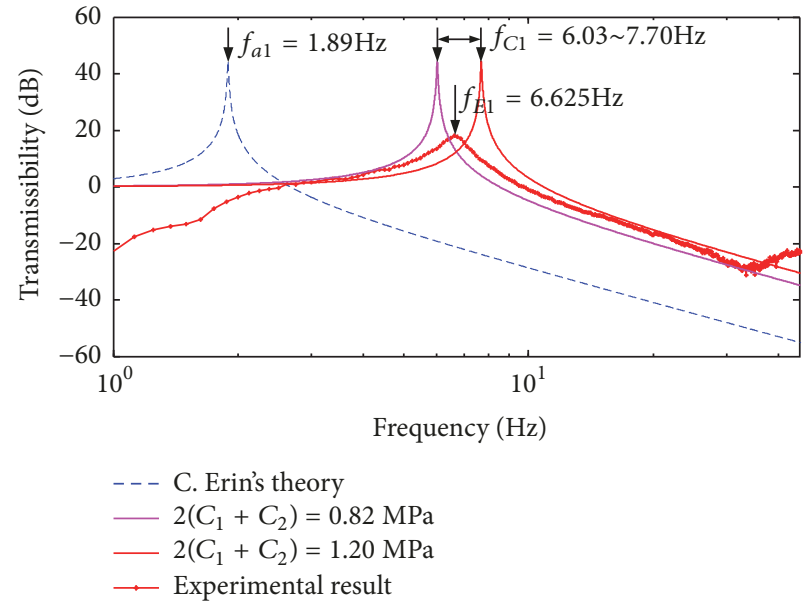

FIGURE 15: Transmissibility curves of experiment 1.

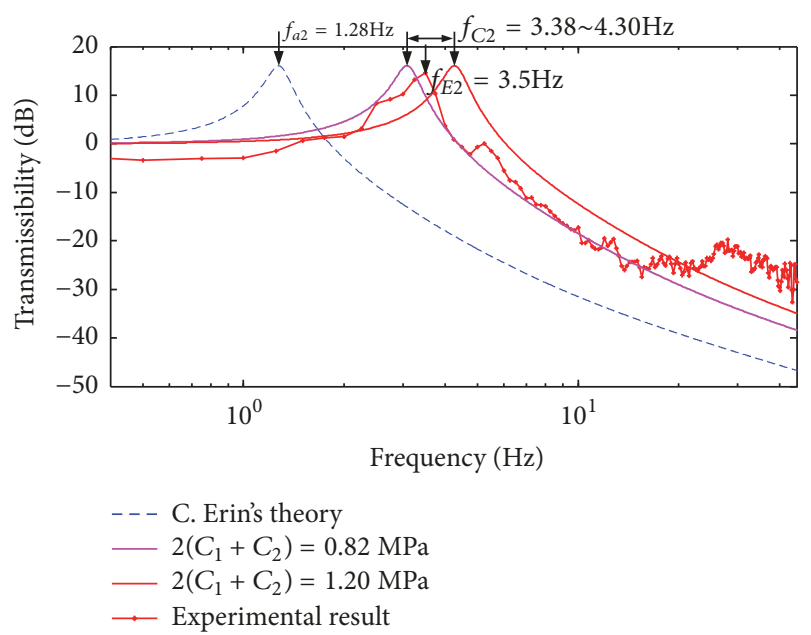

FIGURE 16: Transmissibility curves of experiment 2.

The calculation results of the two structures with different parameters of the dual-chamber pneumatic spring are shown in Table 3. The vertical stiffness, $K_{E 1}$ and $K_{E 2}$, in the experimental tests is within the corresponding ranges of total 
stiffness $K_{\text {total }}$ obtained via calculations, demonstrating the effectiveness of the vertical stiffness model for the elastomeric diaphragm.

\section{Conclusions}

This work developed an analytical model of the elastomeric diaphragm using the Mooney-Rivlin modeling method and elastomeric theory. The stiffness of the elastomeric diaphragm was ignored in the previous model of pneumatic vibration isolators. Specifically, each segmented elastomeric diaphragm can be simplified as uniaxial stretching, and the Mooney-Rivlin model can be adopted to establish the analytical expression of the vertical stiffness. The analytical model of the elastomeric diaphragm is built by combining all segments and can be resolved by numerical solution. The experimental results demonstrated that the analytically predicted behavior is highly similar to the practically observed behavior in terms of the resonant frequency of the isolator after the elastomeric diaphragm model is added to the standard pneumatic cylinder model. Differing from the prior converse correction and experience design, this paper supplies a direct design method that is helpful for optimizing and designing the pneumatic spring parameters in practice.

\section{Appendix}

\section{A.}

See Table 4 .

\section{B. Selected Complex Equations in This Paper}

$$
\begin{aligned}
& K_{1}=\frac{d F_{1}}{d \varepsilon_{1}}=\left\{\frac{4 m g h_{1} d_{0}\left[d_{0} h_{1}+R\left(h_{1}+\varepsilon_{1}\right)\right]}{\left(h_{1}+\varepsilon_{1}\right)^{3}\left[\left(R+2 d_{0}+2 \mathrm{r}-d_{0} h_{3} /\left(h_{3}+\varepsilon_{3}\right)\right)^{2}+\left(h_{1} d_{0} /\left(h_{1}+\varepsilon_{1}\right)+R\right)^{2}\right]^{2}}+4 \pi\left(C_{1}+C_{2}\right)\left(\frac{h_{1}+\varepsilon_{1}}{h_{1}^{2}}\right.\right. \\
& \left.\left.+\frac{h_{1}^{2}}{\left(h_{1}+\varepsilon_{1}\right)^{3}}\right)\right\}\left[\left(R+\frac{h_{1} d_{0}}{h_{1}+\varepsilon_{1}}\right)^{2}-R^{2}\right]-2 \pi\left\{\frac{m g}{\pi\left[\left(R+2 d_{0}+2 r-d_{0} \lambda_{33}\right)^{2}+\left(d_{0} \lambda_{31}+R\right)^{2}\right]}+P_{0}+2\left(C_{1}+C_{2}\right)\right. \\
& \left.\left[\left(\frac{h_{1}+\varepsilon_{1}}{h_{1}}\right)^{2}-\left(\frac{h_{1}}{h_{1}+\varepsilon_{1}}\right)^{2}\right]\right\}\left\{\frac{h_{1} d_{0}\left[d_{0} h_{1}+R\left(h_{1}+\varepsilon_{1}\right)\right]}{\left(h_{1}+\varepsilon_{1}\right)^{3}}\right\} \\
& K_{2}=\frac{d F_{2}}{d \varepsilon_{2}}=2 \pi\left\{4\left(C_{1}+C_{2}\right)\left(\frac{r+\varepsilon_{2}}{r^{2}}+\frac{r^{2}}{\left(r+\varepsilon_{2}\right)^{3}}\right)\left[\frac{\pi}{4} d_{0}\left(\frac{r+\varepsilon_{2}}{r}\right)^{-1}(R+\mathrm{r})-\frac{2}{3} d_{0}\left(\frac{r+\varepsilon_{2}}{r}\right)^{-1} r\right]\right. \\
& -\left[\frac{2 m g}{\pi\left[\left(R+2 d_{0}+2 r-d_{0} h_{3} /\left(h_{3}+\varepsilon_{3}\right)\right)^{2}+\left(d_{0} h_{1} /\left(h_{1}+\varepsilon_{1}\right)+R\right)^{2}\right]}+2\left(C_{1}+C_{2}\right)\left(\left(\frac{r+\varepsilon_{2}}{r}\right)^{2}-\left(\frac{r+\varepsilon_{2}}{r}\right)^{-2}\right)\right] \\
& \cdot\left[\frac{\pi}{4} d_{0}(R+\mathrm{r})-\frac{2}{3} d_{0} r\right] \frac{r}{\left(r+\varepsilon_{2}\right)^{2}}-\frac{2 m g}{\pi\left[\left(R+2 d_{0}+2 \mathrm{r}-d_{0} h_{3} /\left(h_{3}+\varepsilon_{3}\right)\right)^{2}+\left(d_{0} h_{1} /\left(h_{1}+\varepsilon_{1}\right)+R\right)^{2}\right]}\left[\frac{1}{2} d_{0}(R+r)\right. \\
& \left.\left.-\frac{1}{3} d_{0} r\right] \frac{r}{\left(r+\varepsilon_{2}\right)^{2}}\right\} \\
& K_{3}=\frac{d F_{3}}{d \varepsilon_{3}}=\left\{4 \pi\left(C_{1}+C_{2}\right)\left(\frac{h_{3}+\varepsilon_{3}}{h_{3}^{2}}+\frac{h_{3}^{2}}{\left(h_{3}+\varepsilon_{3}\right)^{3}}\right)\right. \\
& \left.-\frac{4 m g h_{3} d_{0}\left[\left(R+2 d_{0}+2 r\right)\left(h_{3}+\varepsilon_{3}\right)-d_{0} h_{3}\right]}{\left(h_{3}+\varepsilon_{3}\right)^{3}\left[\left(R+2 d_{0}+2 r-d_{0} h_{3} /\left(h_{3}+\varepsilon_{3}\right)\right)^{2}+\left(d_{0} h_{1} /\left(h_{1}+\varepsilon_{1}\right)+R\right)^{2}\right]^{2}}\right\}\left[\left(R+2 d_{0}+2 r\right)^{2}-\left(R+2 d_{0}+2 r\right.\right. \\
& \left.\left.-\frac{h_{3} d_{0}}{h_{3}+\varepsilon_{3}}\right)^{2}\right]-2 \pi\left\{P_{0}+\frac{2 m g}{\pi\left[\left(R+2 d_{0}+2 \mathrm{r}-d_{0} h_{3} /\left(h_{3}+\varepsilon_{3}\right)\right)^{2}+\left(d_{0} h_{1} /\left(h_{1}+\varepsilon_{1}\right)+R\right)^{2}\right]}+2\left(C_{1}+C_{2}\right)\right. \\
& \left.\cdot\left[\left(\frac{h_{3}+\varepsilon_{3}}{h_{3}}\right)^{2}-\left(\frac{h_{3}}{h_{3}+\varepsilon_{3}}\right)^{2}\right]\right\}\left[R+2 d_{0}+2 r-\frac{h_{3} d_{0}}{h_{3}+\varepsilon_{3}}\right] \frac{\left(h_{3} d_{0}\right)}{\left(h_{3}+\varepsilon_{3}\right)^{2}}
\end{aligned}
$$




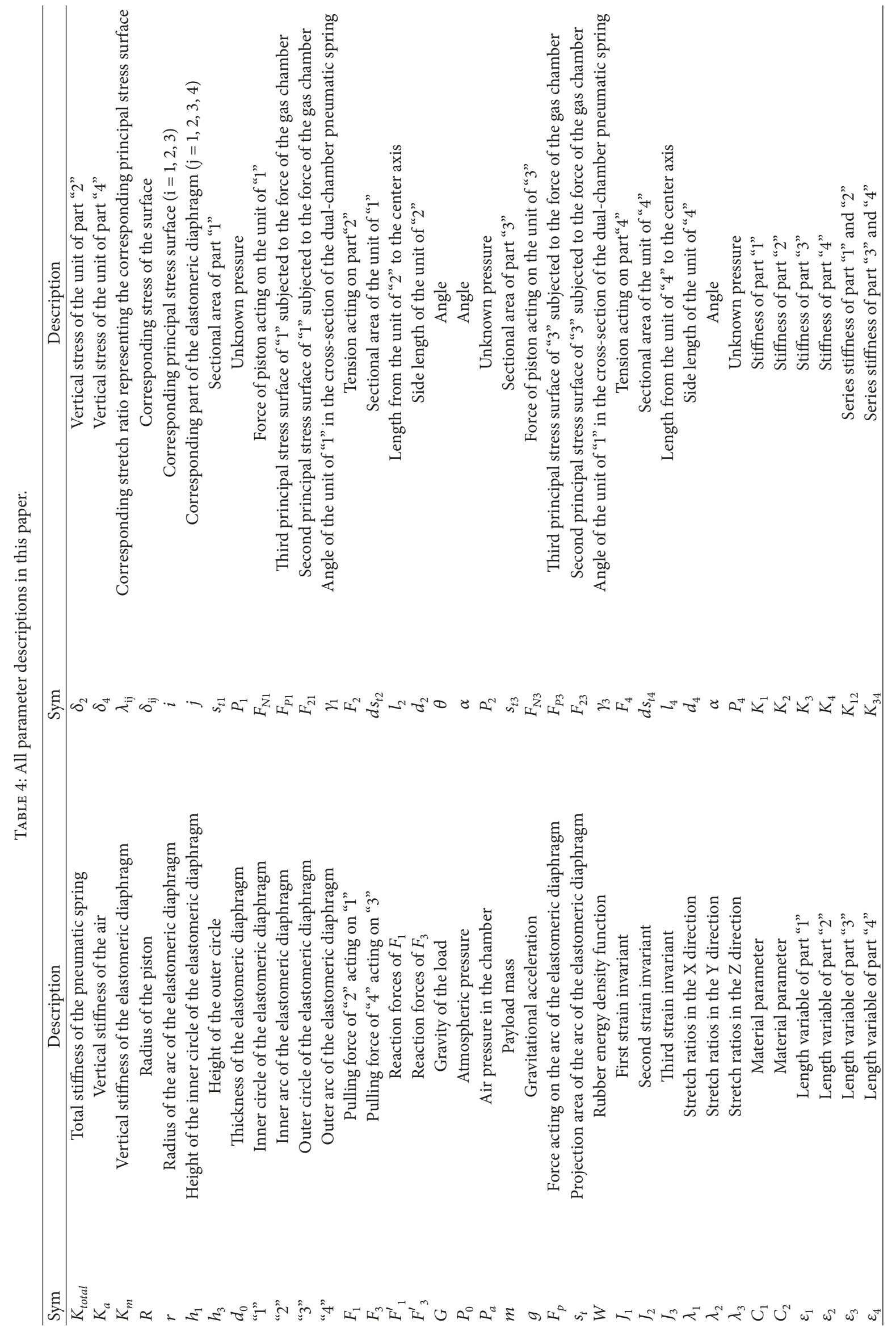




$$
\begin{aligned}
& K_{4}=\frac{d F_{4}}{d \varepsilon_{4}}=2 \pi\left\{4\left(C_{1}+C_{2}\right)\left(\frac{r+\varepsilon_{4}}{r^{2}}+\frac{r^{2}}{\left(r+\varepsilon_{4}\right)^{3}}\right) \cdot\left[\frac{\pi}{4} d_{0}\left(\frac{r+\varepsilon_{4}}{r}\right)^{-1}(\mathrm{R}+r)+\frac{2}{3} d_{0}\left(\frac{r+\varepsilon_{4}}{r}\right)^{-1} r\right]\right. \\
& -\left[\frac{2 m g}{\pi\left[\left(R+2 d_{0}+2 r-d_{0} h_{3} /\left(h_{3}+\varepsilon_{3}\right)\right)^{2}+\left(d_{0} h_{1} /\left(h_{1}+\varepsilon_{1}\right)+R\right)^{2}\right]}+2\left(C_{1}+C_{2}\right)\left(\left(\frac{r+\varepsilon_{4}}{r}\right)^{2}-\left(\frac{r+\varepsilon_{4}}{r}\right)^{-2}\right)\right] \\
& \cdot\left[\frac{\pi}{4} d_{0}(R+r)+\frac{2}{3} d_{0} r\right] \frac{r}{\left(r+\varepsilon_{4}\right)^{2}}-\frac{2 m g}{\pi\left[\left(R+2 d_{0}+2 r-d_{0} h_{3} /\left(h_{3}+\varepsilon_{3}\right)\right)^{2}+\left(d_{0} h_{1} /\left(h_{1}+\varepsilon_{1}\right)+R\right)^{2}\right]}\left[\frac{1}{2} d_{0}(R+r)\right. \\
& \left.\left.+\frac{1}{3} d_{0} r\right] \frac{r}{\left(r+\varepsilon_{4}\right)^{2}}\right\} \\
& \pi\left\{\frac{2 m g}{\pi\left[\left(R+2 d_{0}+2 r-d_{0} h_{3} /\left(h_{3}+\varepsilon_{30}\right)\right)^{2}+\left(d_{0} h_{1} /\left(h_{1}+\varepsilon_{10}\right)+R\right)^{2}\right]}+P_{0}+2\left(C_{1}+C_{2}\right)\left[\left(\frac{h_{1}+\varepsilon_{10}}{h_{1}}\right)^{2}\right.\right. \\
& \left.\left.-\left(\frac{h_{1}}{h_{1}+\varepsilon_{10}}\right)^{2}\right]\right\}\left[\left(R+\frac{h_{1} d_{0}}{h_{1}+\varepsilon_{10}}\right)^{2}-R^{2}\right]=\frac{m g\left[\left(R+2 d_{0}+2 \mathrm{r}-d_{0} h_{3} /\left(h_{3}+\varepsilon_{30}\right)\right)^{2}-\left(d_{0} h_{1} /\left(h_{1}+\varepsilon_{10}\right)+R\right)^{2}\right]}{\left(R+2 d_{0}+2 \mathrm{r}-d_{0} h_{3} /\left(h_{3}+\varepsilon_{30}\right)\right)^{2}+\left(d_{0} h_{1} /\left(h_{1}+\varepsilon_{10}\right)+R\right)^{2}} \\
& 2 \pi\left\{\left[\frac{2 m g}{\pi\left[\left(R+2 d_{0}+2 r-d_{0} h_{3} /\left(h_{3}+\varepsilon_{30}\right)\right)^{2}+\left(d_{0} h_{1} /\left(h_{1}+\varepsilon_{10}\right)+R\right)^{2}\right]}+2\left(C_{1}+C_{2}\right)\left(\left(\frac{r+\varepsilon_{20}}{r}\right)^{2}-\left(\frac{r}{r+\varepsilon_{20}}\right)^{2}\right)\right]\right. \\
& \cdot\left[\frac{\pi d_{0} r(R+\mathrm{r})}{4\left(r+\varepsilon_{20}\right)}-\frac{2 d_{0} r^{2}}{3\left(r+\varepsilon_{20}\right)}\right]+\frac{2 m g}{\pi\left[\left(R+2 d_{0}+2 r-d_{0} h_{3} /\left(h_{3}+\varepsilon_{30}\right)\right)^{2}+\left(d_{0} h_{1} /\left(h_{1}+\varepsilon_{10}\right)+R\right)^{2}\right]}\left[\left(\frac{d_{0} r(R+r)}{\left(r+\varepsilon_{20}\right)}\right)\right. \\
& \left.\left.-\frac{d_{0} r^{2}}{3\left(r+\varepsilon_{20}\right)}\right]\right\}=\frac{m g\left[\left(R+2 d_{0}+2 r-d_{0} h_{3} /\left(h_{3}+\varepsilon_{30}\right)\right)^{2}-\left(d_{0} h_{1} /\left(h_{1}+\varepsilon_{10}\right)+R\right)^{2}\right]}{\left(R+2 d_{0}+2 r-d_{0} h_{3} /\left(h_{3}+\varepsilon_{30}\right)\right)^{2}+\left(d_{0} h_{1} /\left(h_{1}+\varepsilon_{10}\right)+R\right)^{2}} \\
& \pi\left\{\frac{2 m g}{\pi\left[\left(R+2 d_{0}+2 r-d_{0} h_{3} /\left(h_{3}+\varepsilon_{30}\right)\right)^{2}+\left(d_{0} h_{1} /\left(h_{1}+\varepsilon_{10}\right)+R\right)^{2}\right]}+P_{0}+2\left(C_{1}+C_{2}\right)\left[\left(\frac{h_{3}+\varepsilon_{30}}{h_{3}}\right)^{2}\right.\right. \\
& \left.\left.-\left(\frac{h_{3}}{h_{3}+\varepsilon_{30}}\right)^{2}\right]\right\}\left[\left(R+2 d_{0}+2 r\right)^{2}-\left(R+2 d_{0}+2 r-\frac{d_{0} h_{3}}{h_{3}+\varepsilon_{30}}\right)^{2}\right] \\
& =\frac{m g\left[\left(R+2 d_{0}+2 \mathrm{r}-d_{0} h_{3} /\left(h_{3}+\varepsilon_{30}\right)\right)^{2}-\left(d_{0} h_{1} /\left(h_{1}+\varepsilon_{10}\right)+R\right)^{2}\right]}{\left(R+2 d_{0}+2 \mathrm{r}-d_{0} h_{3} /\left(h_{3}+\varepsilon_{30}\right)\right)^{2}+\left(d_{0} h_{1} /\left(h_{1}+\varepsilon_{10}\right)+R\right)^{2}} \\
& 2 \pi\left\{\left[\frac{2 m g}{\pi\left[\left(R+2 d_{0}+2 \mathrm{r}-d_{0} h_{3} /\left(h_{3}+\varepsilon_{30}\right)\right)^{2}+\left(d_{0} h_{1} /\left(h_{1}+\varepsilon_{10}\right)+R\right)^{2}\right]}+2\left(C_{1}+C_{2}\right)\left(\left(\frac{r+\varepsilon_{40}}{r}\right)^{2}-\left(\frac{r}{r+\varepsilon_{40}}\right)^{2}\right)\right]\right. \\
& \cdot\left[\frac{\pi d_{0} r(R+r)}{4\left(r+\varepsilon_{40}\right)}+\frac{2 d_{0} r^{2}}{3\left(r+\varepsilon_{40}\right)}\right] \\
& +\frac{2 m g}{\pi\left[\left(R+2 d_{0}+2 r-d_{0} h_{3} /\left(h_{3}+\varepsilon_{30}\right)\right)^{2}+\left(d_{0} h_{1} /\left(h_{1}+\varepsilon_{10}\right)+R\right)^{2}\right]}\left[\frac{1}{2} d_{0}\left(\frac{r+\varepsilon_{40}}{r}\right)^{-1}(R+r)\right. \\
& \left.\left.+\frac{1}{3} d_{0}\left(\frac{r+\varepsilon_{40}}{r}\right)^{-1} r\right]\right\}=\frac{m g\left[\left(R+2 d_{0}+2 r-d_{0} h_{3} /\left(h_{3}+\varepsilon_{30}\right)\right)^{2}-\left(d_{0} h_{1} /\left(h_{1}+\varepsilon_{10}\right)+R\right)^{2}\right]}{\left(R+2 d_{0}+2 r-d_{0} h_{3} /\left(h_{3}+\varepsilon_{30}\right)\right)^{2}+\left(d_{0} h_{1} /\left(h_{1}+\varepsilon_{10}\right)+R\right)^{2}}
\end{aligned}
$$




\section{Data Availability}

The "Experiment 1.rar" and "Experiment 2.rar" compressed files used to support the findings of this study have been deposited in the Figshare repository (https://figshare.com/s/ 86ee3484e7f439547b71). Open the compressed files and then you can find Erin result, experimental result, experimental data, lower bound for the inherent frequency, and upper bound for the inherent frequency, respectively. You can use MATLAB software to open ".m" and ".fig" type files and use Microsoft Excel software to open ".xlsx" type files.

\section{Conflicts of Interest}

The authors declare that there are no conflicts of interest regarding the publication of this paper.

\section{Acknowledgments}

This work was supported by the National Natural Science Foundation of China under Grant 51475262 and the National Science and Technology Major Project 2015ZX02104003.

\section{References}

[1] J.-W. Liang, H.-Y. Chen, and Q.-W. Wu, "Active suppression of pneumatic vibration isolators using adaptive sliding controller with self-tuning fuzzy compensation," Journal of Vibration and Control, vol. 21, no. 2, pp. 246-259, 2013.

[2] L.-E. Ooi and Z. M. Ripin, "Optimization of an engine mounting system with consideration of frequency-dependent stiffness and loss factor," Journal of Vibration and Control, vol. 22, no. 10, pp. 2406-2419, 2014.

[3] F. C. Nelson, "Vibration isolation: a review, I. Sinusoidal and random excitations," Shock and Vibration, vol. 1, no. 5, pp. 485493, 1994.

[4] F. C. Nelson, "Vibration Isolation Review: IL Shock Excitation," Shock and Vibration, vol. 3, no. 6, pp. 451-459, 1996.

[5] C. M. Harris, Harris' Shock and Vibration Handbook, McGrawHill, New York, NY, USA, 2002.

[6] D. B. DeBra and J. Bryan, "Design of laminar flow restrictors for damping pneumatic vibration isolators," CIRP Annals Manufacturing Technology, vol. 33, no. 1, pp. 351-356, 1984.

[7] C. Erin, B. Wilson, and J. Zapfe, "An improved model of a pneumatic vibration isolator: theory and experiment," Journal of Sound and Vibration, vol. 218, no. 1, pp. 81-101, 1998.

[8] J.-H. Lee and K.-J. Kim, "Modelling of nonlinear complex stiffness of dual-chamber pneumatic spring for precision vibration isolations," Journal of Sound and Vibration, vol. 301, no. 3-5, pp. 909-926, 2007.

[9] J.-H. Lee and K.-J. Kim, "A method of transmissibility design for dual-chamber pneumatic vibration isolator," Journal of Sound and Vibration, vol. 323, no. 1-2, pp. 67-92, 2009.

[10] $\mathrm{H}$. $\mathrm{Pu}, \mathrm{X}$. Luo, and $\mathrm{X}$. Chen, "Modeling and analysis of dual-chamber pneumatic spring with adjustable damping for precision vibration isolation," Journal of Sound and Vibration, vol. 330, no. 15, pp. 3578-3590, 2011.

[11] T. Mankovits and T. Szabó, "Finite element analysis of rubber bumper used in air-springs," Procedia Engineering, vol. 48, pp. 388-395, 2012.
[12] Y. Zhu, Q. Li, D. Xu, C. Hu, and M. Zhang, "Modeling and analysis of a negative stiffness magnetic suspension vibration isolator with experimental investigations," Review of Scientific Instruments, vol. 83, no. 9, pp. 95-108, 2012.

[13] Q. Li, Research on an innovative vibration isolator with magnetic suspension positive stiffness combined with rubber negative stiffness system [Ph.D. thesis], Tsinghua University, Beijing, China, 2013.

[14] S. S. Rao, Mechanical Vibrations, Tsinghua University Press, Beijing, China, 2009.

[15] L. R. G. Treloar, The Physics of Rubber Elasticity, Oxford University Press, Oxford, UK, 1975.

[16] A. N. Gent, Engineering with Rubber-How to Design Rubber Components, Hanser Publishers, Munich, Germany, 2001.

[17] http://www.mfopt.com/products_detail/productId=2688.html. 


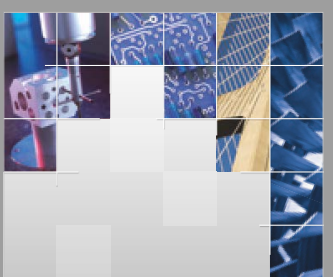

\section{Enfincering}
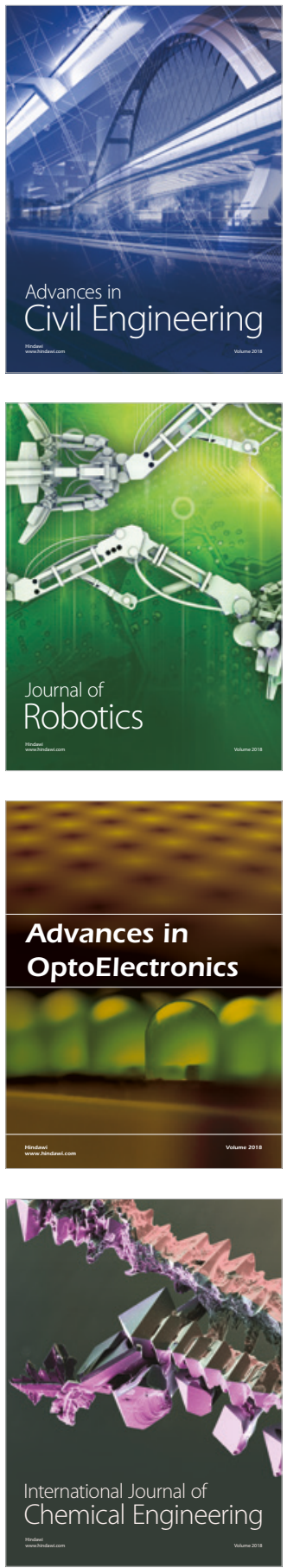

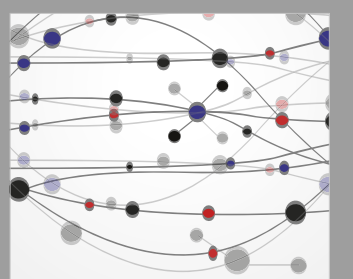

\section{Rotating \\ Machinery}

The Scientific World Journal

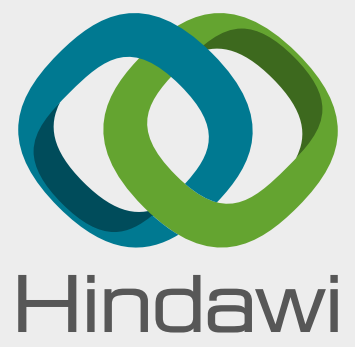

Submit your manuscripts at

www.hindawi.com
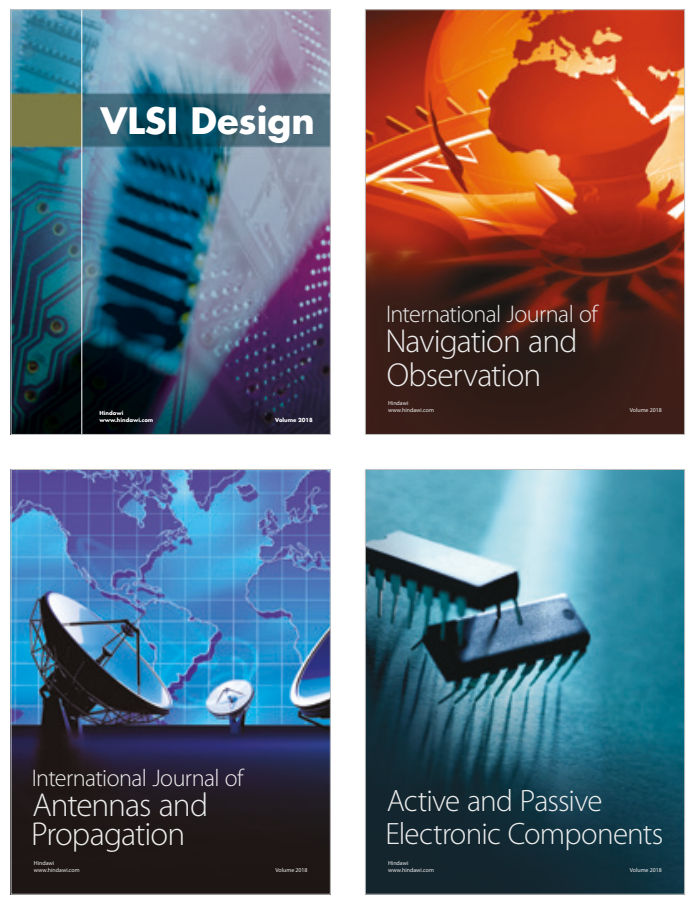
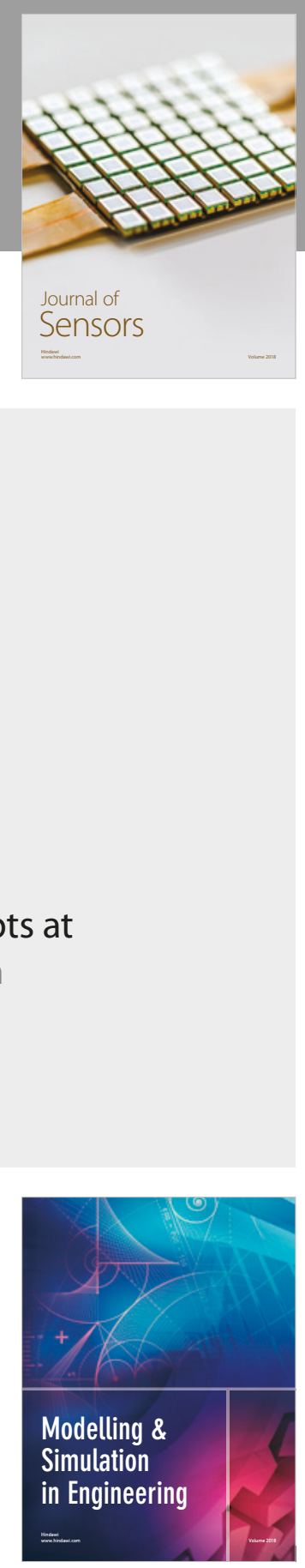

\section{Advances \\ Multimedia}
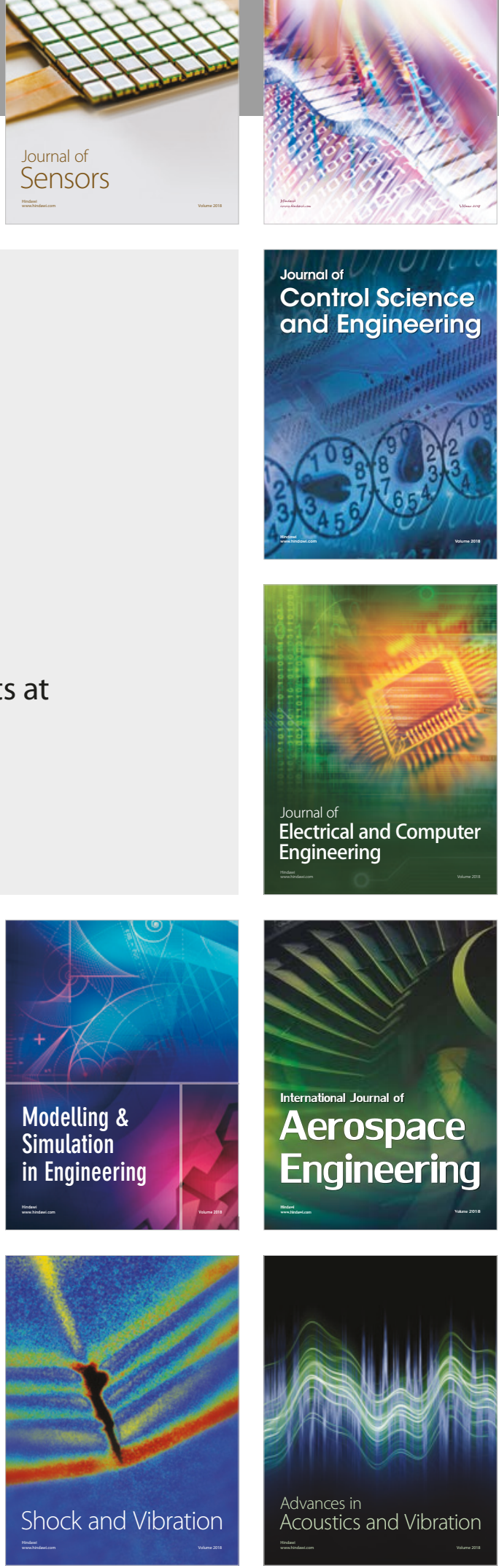